\title{
QUANTUM EULER-POISSON SYSTEMS: GLOBAL EXISTENCE AND EXPONENTIAL DECAY
}

BY

ANSGAR JÜNGEL (Fachbereich Mathematik und Statistik, Universität Konstanz, Fach D193, 78457 Konstanz, Germany)

AND

HAILIANG LI (Institut für Mathematik, Universität Wien, 1090 Vienna, Austria and Department of Pure and Applied Mathematics, Graduate School of Information Science and Technology, Osaka University, Toyonaka, Osaka 560-0043, Japan)

Abstract. A one-dimensional transient quantum Euler-Poisson system for the electron density, the current density, and the electrostatic potential in bounded intervals is considered. The equations include the Bohm potential accounting for quantum mechanical effects and are of dispersive type. They are used, for instance, for the modelling of quantum semiconductor devices.

The existence of local-in-time solutions with small initial velocity is proven for general pressure-density functions. If a stability condition related to the subsonic condition for the classical Euler equations is imposed, the local solutions are proven to exist globally in time and tend to the corresponding steady-state solution exponentially fast as the time tends to infinity.

\section{Introduction.}

1.1. The Model Equations. In 1927, Madelung gave a fluid-dynamical description of quantum systems governed by the linear Schrödinger equation for the wave function $\psi$ :

$$
\begin{aligned}
i \varepsilon \partial_{t} \psi & =-\frac{\varepsilon^{2}}{2} \Delta \psi-V \psi \quad \text { in } \mathbb{R}^{d} \times(0, \infty), \\
\psi(\cdot, 0) & =\psi_{0} \quad \text { in } \mathbb{R}^{d}
\end{aligned}
$$

Received November 6, 2003.

2000 Mathematics Subject Classification. Primary 35A07, 35B40.

Key words and phrases. Quantum Euler-Poisson system, existence of global-in-time classical solutions, nonlinear fourth-order wave equation, exponential decay rate, long-time behavior of the solutions.

E-mail address: juengel@mathematik.uni-mainz.de

Current address: Fachbereich Mathematik und Informatik, Johannes Gutenberg-Universität, 55099 Mainz, Germany.

E-mail address: lihl@math.sci.osaka-u.ac.jp

Current address: Department of Mathematics, Capital Normal University, Beijing 100037, P. R. China. 
where $d \geq 1$ is the space dimension, $\varepsilon>0$ denotes the scaled Planck constant, and $V=V(x, t)$ is some (given) potential. Separating the amplitude and phase of $\psi=$ $|\psi| \exp (i S / \varepsilon)$, the particle density $\rho=|\psi|^{2}$, and the particle current density $j=\rho \nabla S$ for irrotational flow satisfy the so-called Madelung equations [21]

$$
\begin{gathered}
\partial_{t} \rho+\operatorname{div} j=0, \\
\partial_{t} j+\operatorname{div}\left(\frac{j \otimes j}{\rho}\right)-\rho \nabla \phi-\frac{\varepsilon^{2}}{2} \rho \nabla\left(\frac{\Delta \sqrt{\rho}}{\sqrt{\rho}}\right)=0 \quad \text { in } \mathbb{R}^{d} \times(0, \infty),
\end{gathered}
$$

where the $i$-th component of the convective term $\operatorname{div}(j \otimes j / \rho)$ equals

$$
\sum_{k=1}^{d} \frac{\partial}{\partial x_{k}}\left(\frac{j_{i} j_{k}}{\rho}\right) .
$$

Equations (1)-(2) can be interpreted as the pressureless Euler equations including the quantum Bohm potential

$$
\frac{\varepsilon^{2}}{2} \frac{\Delta \sqrt{\rho}}{\sqrt{\rho}} .
$$

They have been used for the modeling of superfluids like Helium II [16, 20].

Recently, Madelung-type equations have been derived to model quantum phenomena in semiconductor devices, like resonant tunneling diodes, starting from the WignerBoltzmann equation [6] or from a mixed-state Schrödinger-Poisson system [8, 9]. There are several advantages of the fluid-dynamical description of quantum semiconductors. First, kinetic equations, like the Wigner equation or Schrödinger systems, are computationally very expensive, whereas for Euler-type equations, efficient numerical algorithms are available $[5,25]$. Second, the macroscopic description allows for a coupling of classical and quantum models. Indeed, setting the Planck constant $\varepsilon$ in (2) equal to zero, we obtain the classical pressureless equations, so in both pictures, the same (macroscopic) variables can be used. Finally, as semiconductor devices are modeled in bounded domains, it is easier to find physically relevant boundary conditions for the macroscopic variables than for the Wigner function or for the wave function.

The Madelung-type equations derived by Gardner [6] and Gasser et al. [8] also include a pressure term and a momentum relaxation term taking into account interactions of the electrons with the semiconductor crystal, and are self-consistently coupled to the Poisson equation for the electrostatic potential $\phi$ :

$$
\begin{gathered}
\partial_{t} \rho+\operatorname{div} j=0, \\
\partial_{t} j+\operatorname{div}\left(\frac{j \otimes j}{\rho}\right)+\nabla P(\rho)-\rho \nabla \phi-\frac{\varepsilon^{2}}{2} \rho \nabla\left(\frac{\Delta \sqrt{\rho}}{\sqrt{\rho}}\right)=-\frac{j}{\tau}, \\
\lambda^{2} \Delta \phi=\rho-\mathcal{C}(x) \quad \text { in } \Omega \times(0, \infty),
\end{gathered}
$$

where $\Omega \subset \mathbb{R}^{d}$ is a bounded domain, $\tau>0$ is the (scaled) momentum relaxation time constant, $\lambda>0$ is the (scaled) Debye length, and $\mathcal{C}(x)$ is the doping concentration modelling the semiconductor device under consideration $[12,24]$. The pressure is assumed 
to depend only on the particle density and, like in classical fluid dynamics, often the expression

$$
P(\rho)=\frac{T_{0}}{\gamma} \rho^{\gamma}, \quad \rho \geq 0, \quad \gamma \geq 1,
$$

with the temperature constant $T_{0}>0$, is employed [6, 11]. Isothermal fluids correspond to $\gamma=1$, isentropic fluids to $\gamma>1$. Notice that the particle temperature is $T(\rho)=T_{0} \rho^{\gamma-1}$. In this paper we consider general (smooth) pressure functions. Equations (4)-(6) are referred to as the quantum Euler-Poisson system or as the quantum hydrodynamic model.

In this paper, we investigate the (local and global) existence and long-time behavior of solutions of the following one-dimensional quantum Euler-Poisson system:

$$
\begin{aligned}
\rho_{t}+j_{x} & =0, \\
j_{t}+\left(\frac{j^{2}}{\rho}+P(\rho)\right)_{x} & =\rho \phi_{x}+\frac{1}{2} \varepsilon^{2} \rho\left(\frac{(\sqrt{\rho})_{x x}}{\sqrt{\rho}}\right)_{x}-\frac{j}{\tau}, \\
\phi_{x x} & =\rho-\mathcal{C}(x),
\end{aligned}
$$

with the following initial and boundary conditions:

$$
\begin{gathered}
\rho(x, 0)=\varrho_{1}(x)>0, \quad j(x, 0)=j_{1}(x)=: \varrho_{1}(x) v_{1}(x), \\
\rho(0, t)=\rho_{1}, \quad \rho(1, t)=\rho_{2}, \quad \rho_{x}(0, t)=\rho_{x}(1, t)=0, \\
\phi(0, t)=0, \quad \phi(1, t)=\Phi_{0},
\end{gathered}
$$

for $(x, t) \in(0,1) \times(0, \infty)$, where $\rho_{1}, \rho_{2}, \Phi_{0}>0$, and $v_{1}$ is the initial velocity.

The existence and uniqueness of steady-state (classical) solutions to the quantum Euler-Poisson system for current density $j_{0}=0$ (thermal equilibrium) has been studied in $[1,7]$. The stationary equations for $j_{0}>0$ have been considered in $[4,11,27]$ for general monotone pressure functions, however, with different boundary conditions, assuming Dirichlet data for the velocity potential $S$ [11] or employing nonlinear boundary conditions $[4,27]$. Existence of steady-state solutions to (8)-(10) subject to the boundary conditions (12)--(13) is proven in [10] for the linear pressure function $P(\rho)=\rho$ and in [14] for general pressure functions $P(\rho)$ also allowing for non-convex or non-monotone pressure-density relations. So far, to our knowledge, the only known results on the existence of the time-dependent system (4)-(6) have been obtained in [13] for smooth local-in-time solutions on bounded domains and in [17] for "small" irrotational globalin-time solutions in the whole space assuming strictly convex pressure functions and a constant doping profile.

In the present paper, we consider the initial-boundary-value problem (IBVP) 8-13 for general pressure and non-constant doping profile, and we focus on the local and global existence of classical solutions $(\rho, j, \phi)$ of the IBVP 8-13 and their time-asymptotic convergence to the stationary solutions $\left(\rho_{0}, j_{0}, \phi_{0}\right)$ obtained in [14]. First, we show that there exists a classical local-in-time solution for regular initial data. Second, we prove that if a certain "subsonic" condition (see 25) holds and if the initial data is a perturbation of a stationary solution $\left(\rho_{0}, j_{0}, \phi_{0}\right)$, a classical solution $(\rho, j, \phi)$ exists globally in time and tends to $\left(\rho_{0}, j_{0}, \phi_{0}\right)$ exponentially fast as time tends to infinity. 
In dealing with the IBVP 8.13 we have to overcome the following difficulties. First, since the general pressure $P(\rho)$ can be non-convex (even zero or "negative"; see Remark 1.6), the left part of Eqs. 810 may be not hyperbolic any more. Unlike [17], we cannot apply the local existence theory of quasilinear symmetric hyperbolic systems $[3,15,22,23]$. We have to establish a new local existence theory. Second, the appearance of the nonlinear quantum Bohm potential in 9 requires that the density is strictly positive for regular solutions. This together with the structure of the quantum term causes problems in the local and global existence proofs.

1.2. Main results. Before stating our main results we introduce some notation. We denote by $L^{2}=L^{2}(0,1)$ and $H^{k}=H^{k}(0,1)$ the Lebesgue space of square integrable functions and the Sobolev space of functions with square integrable weak derivatives of order $k$, respectively. The norm of $L^{2}$ is denoted by $\|\cdot\|_{0}=\|\cdot\|$, and the norm of $H^{k}$ is $\|\cdot\|_{k}$. The space $H_{0}^{k}=H_{0}^{k}(0,1)$ is the closure of $C_{0}^{\infty}(0,1)$ in the norm of $H^{k}$. Let $T>0$ and let $\mathcal{B}$ be a Banach space. Then $C^{k}(0, T ; \mathcal{B})\left(C^{k}([0, T] ; \mathcal{B})\right.$, respectively) denotes the space of $\mathcal{B}$-valued $k$-times continuously differentiable functions on $(0, T)([0, T]$, respectively), $L^{2}(0, T ; \mathcal{B})$ is the space of $\mathcal{B}$-valued $L^{2}$-functions on $(0, T)$, and $W^{k, p}(0, T ; \mathcal{B})$ the space of $\mathcal{B}$-valued $W^{k, p}$-functions on $(0, T)$. Finally, $C$ always denotes a generic positive constant.

It is convenient to make use of the variable transformation $\rho=\omega^{2}$ in $8-10$, which yields the following IBVP for $(\omega, j, \phi)$ :

$$
\begin{aligned}
2 \omega \omega_{t}+j_{x} & =0 \\
j_{t}+\left(\frac{j^{2}}{\omega^{2}}+P\left(\omega^{2}\right)\right)_{x} & =\omega^{2} \phi_{x}+\frac{1}{2} \varepsilon^{2} \omega^{2}\left(\frac{\omega_{x x}}{\omega}\right)_{x}-\frac{j}{\tau}, \\
\phi_{x x} & =\omega^{2}-\mathcal{C}(x)
\end{aligned}
$$

with the initial and boundary conditions

$$
\begin{gathered}
(w, j)(x, 0)=\left(\omega_{1}, j_{1}\right)(x)=\left(\sqrt{\varrho_{1}}, \varrho_{1} v_{1}\right)(x), \\
w(0, t)=\sqrt{\rho_{1}}, \quad w(1, t)=\sqrt{\rho_{2}}, \quad w_{x}(0, t)=w_{x}(1, t)=0, \\
\phi(0, t)=0, \quad \phi(1, t)=\Phi_{0},
\end{gathered}
$$

for $x \in(0,1), t \geq 0$. This problem is equivalent to $8-13$ for classical solutions with positive particle density.

We will assume throughout this paper compatibility conditions for the IBVP 14-19 in the sense that the time derivatives of the boundary values and the spatial derivatives of the initial data are compatible at $(x, t)=(0,0)$ and $(x, t)=(1,0)$ in $14-16$. We will prove the following local existence result for the IBVP 14-19:

TheOrem 1.1. Assume that

$$
P \in C^{4}(0,+\infty), \quad \mathcal{C} \in H^{2}
$$

$\left(\omega_{1}, j_{1}\right) \in H^{6} \times H^{5}$ such that $\omega_{1}(x)>0$ for $x \in[0,1]$, and for some $\alpha \in\left[(1+2 \sqrt{2} \varepsilon)^{-1}, 1\right)$

$$
\left\|v_{1}\right\|_{C^{1}([0,1])}<\frac{(1-\alpha) \omega_{*}}{8 \sqrt{2}\left\|\omega_{1}\right\|_{1}},
$$


where

$$
\omega_{*}=\min _{x \in[0,1]} \omega_{1}(x)>0 .
$$

Then, there is a number $T_{* *}$ (determined by 128), such that there exists a unique classical solution $(\omega, j, \phi)$ of the IBVP $(14)-(19)$ in the time interval $[0, T]$, with $0<T \leq T_{* *}$, satisfying $\omega \geq(1-\alpha) \omega_{*}>0$ in $[0,1] \times[0, T]$ and

$$
\|\omega(t)\|_{6}^{2}+\|j(t)\|_{5}^{2}+\|\phi(t)\|_{4}^{2}<\infty \quad \text { for } t \leq T .
$$

REMARK 1.2. (1) It is well known that for classical hydrodynamic equations, monotone pressure-density relations are required to guarantee short-time existence of classical solutions $[2,18]$. The condition 20 means that this condition is not necessary (to a certain extent) when the quantum effects are taken into account.

(2) Condition 21 is needed to prove the positivity of the particle density. A similar condition has been used to prove the existence of stationary solutions [11]. This condition allows, for arbitrarily large current densities $j_{1}=w_{1}^{2} v_{1}$, for instance, if $w_{1}$ is a sufficiently large constant.

(3) We are able to show the statements of Theorem 1.1 under the slightly more general condition

$$
\left\|v_{1}\right\|_{C^{1}([0,1])}<\min \left\{\alpha \varepsilon, \frac{(1-\alpha)}{2 \sqrt{2}}\right\} \frac{\omega_{*}}{4\left\|\omega_{1}\right\|_{1}}, \quad \alpha \in(0,1) .
$$

Then 21 is a special case for $\alpha>(1+2 \sqrt{2} \varepsilon)^{-1}$ which is equivalent to $\alpha \varepsilon \geq(1-\alpha) / 2 \sqrt{2}$.

(4) The local existence of the Cauchy problem in $\mathbb{R}^{d}$ or $\mathbb{T}^{d}$ can be shown in the same framework; see [19].

Theorem 1.1 is proven by an iteration method and compactness arguments. More precisely, we construct a sequence of approximate solutions that is uniformly bounded in a certain Sobolev space in a fixed (maybe small) time interval. Compactness arguments then imply that there is a limiting solution which proves to be a local-in-time solution of 14-19. Unlike [17], we cannot apply the theory of quasilinear symmetric hyperbolic systems $[3,15,22,23]$ to construct (local) approximate solutions and obtain uniform bounds in Sobolev spaces because the pressure can be non-convex, causing the loss of entropy and hyperbolicity of 14-15.

The idea of the local existence result is first to linearize the system 14-16 around its initial state $\left(\omega_{1}, j_{1}, \phi_{1}\right)$, where $\phi_{1}$ solves the Dirichlet problem 16 and 19 with $\omega$ replaced by $\omega_{1}$, and to consider the equations for the perturbation $(\psi, \eta, e)=\left(\omega-\omega_{1}, j-j_{1}, \phi-\phi_{1}\right)$. The main idea is to write the evolution equation for the perturbed particle density as a semilinear fourth-order wave equation. Then, we construct approximate solutions $\left(\psi_{i}, \eta_{i}, e_{i}\right)(i \geq 1)$ from a fixed-point procedure, which are expected to converge to a solution $(\psi, \eta, e)$ of the perturbed problem as $i \rightarrow \infty$. For this, we derive uniform bounds in Sobolev spaces on a uniform time interval and apply standard compactness arguments ,(see Sec. 3). A further analysis shows that $(\omega, j, \phi)=\left(\omega_{1}+\psi, j_{1}+\eta, \phi_{1}+e\right)$ with $\omega>0$ is the expected local (in time) solution of the original problem 14-19.

To extend the local classical solution globally in time, we need to establish uniform estimates. We consider the situation when the initial data is close to the stationary 
solution $\left(\omega_{0}, j_{0}, \phi_{0}\right)$ of $(14) \cdot(16)$ with boundary conditions 18-19. The existence of stationary solutions $\left(\omega_{0}, j_{0}, \phi_{0}\right)$ of the boundary-value problem (14)-(16) and 18-19 for general pressure functions $P(\rho)$ was obtained in [14] (sce Theorem 1.3 below).

Assume that there is a function $\mathcal{A} \in H^{2}(0,1)$ satisfying

$$
\mathcal{A}(x)>0 \text { for } x \in(0,1) . \quad \mathcal{A}(0)=\rho_{1} . \quad \mathcal{A}(1)=\rho_{2}, \quad \mathcal{A}_{x}(0)=\mathcal{A}_{x}(1)=0
$$

such that for a set $E \subseteq[0,1]$, it holds

$$
P^{\prime}(\mathcal{A})-\frac{j_{0}^{2}}{\mathcal{A}^{2}} \begin{cases}\leq 0, & x \in E, \\ >0, & x \in[0,1] \backslash E .\end{cases}
$$

Then we conclude the existence of stationary solutions $\left(w_{0}, j_{0}, \phi_{0}\right)$ of $14-16$ satisfying the boundary conditions 18-19:

Theorem 1.3 ([14]). Let 20, 23-24 hold. For given $\kappa \in(0,1)$, assume that

$$
\min _{x \in[0,1]} \mathcal{A}^{2}\left(\frac{1}{4} \kappa \varepsilon^{2}+P^{\prime}(\mathcal{A})\right)>j_{0}^{2} .
$$

Then there is a unique solution $\left(\omega_{0}, j_{0}, \phi_{0}\right)$ of the stationary version of the boundary-value problem $14-16$ and $18-19$ such that

$$
\mathcal{A}_{*}\left\|w_{0}-\sqrt{\mathcal{A}}\right\|^{2}+A_{0}\left\|w_{0 x}\right\|_{3}^{2}+\left\|\phi_{0 x}\right\|_{1}^{2} \leq C \delta_{0},
$$

provided $\delta_{0}:=\left\|\mathcal{A}^{\prime}\right\|_{1}+\|\mathcal{A}-\mathcal{C}\|$ is small enough. Here, $\mathcal{A}_{*}=\min _{x \in[0,1]} \mathcal{A}(x)$,

$$
A_{0}=\min _{x \in[0,1]}\left(\frac{1}{4} \kappa \varepsilon^{2}+P^{\prime}(\mathcal{A})-j_{0}^{2} \mathcal{A}^{-2}\right)>0,
$$

and $C>0$ is a constant depending on $j_{0}, \tau$, and $\mathcal{A}$.

Let $\rho_{0}=\omega_{0}^{2}$. Then $\left(\rho_{0}, j_{0}, \phi_{0}\right)$ is a solution of the stationary version of the boundaryvalue problem $8-10$ and $12-13$ satisfying

$$
\mathcal{A}_{*}\left\|\rho_{0}-\mathcal{A}\right\|^{2}+A_{0}\left\|\rho_{0 x}\right\|_{3}^{2}+\left\|\phi_{0 x}\right\|_{1}^{2} \leq C^{\prime} \delta_{0},
$$

and $C^{\prime}>0$ is a constant depending on $j_{0}, \tau$, and $\mathcal{A}$.

REMARK 1.4. (1) When $E=\emptyset$ assumption 24 corresponds exactly to the subsonic condition for classical fluids $[2,18]$. We recall that a classical fluid is in the subsonic state if the velocity is smaller than the sound speed $\sqrt{P^{\prime}(\rho)}$. Only for subsonic fluids, we can expect to have existence of classical solutions $[2,18]$. Therefore, in order to get existence of classical solutions of the quantum hydrodynamic equations, we expect that a condition corresponding to the classical subsonic condition is needed. It turns out that 24 is such a condition. Notice that condition 25 can allow for non-empty sets $E$ when quantum effects are involved.

(2) Condition 24 can be replaced by

$$
\frac{1}{4} \kappa \varepsilon^{2}+|E| \min _{x \in E}\left(p^{\prime}(\mathcal{A})-j_{0}^{2} \mathcal{A}^{-2}\right)>0, \quad \kappa \in(0,1),
$$

in order to obtain the existence and uniqueness of classical solutions. Here, $|E|$ denotes the volume of the subset $E$. 
(3) We recall that in the steady state, the current density $j_{0}$ is a constant. If $j_{0}=0$, we obtain the thermal equilibrium state. Condition 24 is satisfied if $j_{0}>0$ is sufficiently small. Thus, Theorem 1.3 means that we can show the existence of solutions "close" to the thermal equilibrium state.

In the following, we use the abbreviation

$$
\psi_{0}=\omega_{1}-\omega_{0}, \quad \eta_{0}=j_{1}-j_{0} .
$$

In view of the uniform a-priori estimates of Sec. 2, we are able to extend the local classical solution globally in time and prove its exponential convergence to the stationary solution $\left(w_{0}, j_{0}, \phi_{0}\right)$ :

Theorem 1.5. Assume that $20,23-25$ hold. Let $\left(\omega_{0}, j_{0}, \phi_{0}\right)$ be the stationary solution of the boundary-value problem 14-16 and 18-19 given by Theorem 1.3 for sufficiently small $\delta_{0}$. Assume that the initial datum $\left(\omega_{1}, j_{1}\right) \in H^{6} \times H^{5}$ satisfies 21 and $\omega_{1}>0$ in $[0,1]$. Then there is a number $m_{1}>0$ such that if

$$
\left\|\psi_{0}\right\|_{6}+\left\|\eta_{0}\right\|_{5}=\left\|w_{1}-w_{0}\right\|_{6}+\left\|j_{1}-j_{0}\right\|_{5} \leq m_{1},
$$

the (classical) solution $(\omega, j, \phi)$ of the IBVP (14)-(19) exists globally in time and satisfies

$$
\left\|\left(\omega-\omega_{0}\right)(t)\right\|_{6}^{2}+\left\|\left(j-j_{0}\right)(t)\right\|_{5}^{2}+\left\|\left(\phi-\phi_{0}\right)(t)\right\|_{4}^{2} \leq C\left(\left\|\psi_{0}\right\|_{6}^{2}+\left\|\eta_{0}\right\|_{5}^{2}\right) e^{-\Lambda_{0} t}
$$

for all $t \geq 0$. Here, $C>0$ and $\Lambda_{0}>0$ are constants independent of the time variable $t$.

REMARK 1.6. Theorems 1.1-1.5 also apply for non-monotone or even "negative" pressure functions. These functions are related to quantum mechanical phenomena in which the motion of the particles is affected by their attractive interaction [16]. A typical example is the focusing nonlinear Schrödinger equation. In fact, this equation is formally equivalent to the quantum Euler-Poisson system with infinite relaxation time and with "negative" pressure.

Using Theorems 1.1-1.5 and the variable transformation $\rho=\omega^{2}$, we also obtain the local and global existence of classical solutions of the original IBVP 8-13 and can establish their large-time behavior:

Theorem 1.7. Let 20 hold. Assume that $\left(\sqrt{\varrho_{1}}, j_{1}\right) \in H^{6} \times H^{5}$ such that $\varrho_{1}>0$ in $[0,1]$ and

where

$$
\left\|v_{1}\right\|_{C^{1}([0,1])}<\min \left\{\alpha \varepsilon, \frac{(1-\alpha)}{2 \sqrt{2}}\right\} \frac{\varrho_{*}}{4\left\|\sqrt{\varrho}_{1}\right\|_{1}}, \quad \alpha \in(0,1),
$$

$$
\varrho_{*}=\min _{x \in[0,1]} \sqrt{\varrho}_{1}(x) .
$$

Then there is a number $T_{*}^{\prime}>0$ such that there exists a classical solution $(\rho, j, \phi)$ of the IBVP (8)-(13) in $t \in\left[0, T_{*}^{\prime}\right]$ satisfying $\rho>0$ in $[0,1] \times\left[0, T_{*}^{\prime}\right]$ and

$$
\|\rho(t)\|_{6}^{2}+\|j(t)\|_{5}^{2}+\|\phi(t)\|_{4}^{2}<\infty, \quad t \leq T_{*}^{\prime} .
$$

Furthermore, assume that 23-25 hold and let $\left(\rho_{0}, j_{0}, \phi_{0}\right)$ be the stationary solution of the boundary-value problem $8-10$ and $12-13$ given by Theorem 1.3 with sufficiently 
small $\delta_{0}$. Then, there is a number $m_{2}>0$ such that if $\left\|\sqrt{\varrho_{1}}-\sqrt{\rho_{0}}\right\|_{6}+\left\|\eta_{0}\right\|_{5} \leq m_{2}$, the solution $(\rho, j, \phi)$ of the IBVP (14)-(19) exists globally in time and satisfies

$$
\left\|\left(\rho-\rho_{0}\right)(t)\right\|_{6}^{2}+\left\|\left(j-j_{0}\right)(t)\right\|_{5}^{2}+\left\|\left(\phi-\phi_{0}\right)(t)\right\|_{4}^{2} \leq C\left(\left\|\psi_{0}\right\|_{6}^{2}+\left\|\eta_{0}\right\|_{5}^{2}\right) e^{-\Lambda_{1} t},
$$

for all $t \geq 0$, where $C>0$ and $\Lambda_{1}>0$ are constants independent of $t$ and the pair $\left(\psi_{0}, \eta_{0}\right)$ is defined in 28 .

This paper is arranged as follows. Section 2 is concerned with uniform a-priori estimates of local (in time) solutions. We reformulate the original problem in Sec. 2.1 as a nonlinear fourth-order wave equation and establish the a-priori estimates for local solutions in Sec. 2.2. The a-priori estimates and the local existence result of Sec. 3 imply the global existence. In order to prove the local existence result, we first give a result on the existence of solutions of an abstract fourth-order wave equation (Sec. 3.1). This wave equation allows us to construct a sequence of approximate solutions converging to a local solution of the problem under consideration (Sec. 3.2).

2. Proof of Theorem 1.5. In this section, we establish uniform a-priori estimates for local classical solutions of 14-16. This yields, together with the usual continuity argument, the existence of global-in-time solutions and proves Theorem 1.5. For notational simplicity, we set $\tau=1$.

2.1. Reformulation of the original problem. Let $\left(\omega_{0}, j_{0}, \phi_{0}\right)$ be the steady-state solution of the boundary-value problem $14-16$ and 18-19. For any $T>0$, assume that $(\omega, j, \phi)$ is a solution to the IBVP $14-19$ in $[0, T]$.

Differentiating 14 with respect to $t$ and 15 with respect to $x$ and adding the resulting equations leads to a nonlinear fourth-order wave equation for $w$ :

$$
\omega_{t t}+\omega_{t}+\frac{1}{\omega} \omega_{t}^{2}+\frac{1}{2 \omega}\left(\omega^{2} \phi_{x}\right)_{x}-\frac{1}{2 \omega}\left[P\left(\omega^{2}\right)+\frac{j^{2}}{\omega^{2}}\right]_{x x}+\frac{1}{4} \varepsilon^{2} \omega_{x x x x}-\frac{1}{4} \varepsilon^{2} \frac{\omega_{x x}^{2}}{\omega}=0,
$$

where we have used the identity

$$
\left[\omega^{2}\left(\frac{\omega_{x x}}{\omega}\right)_{x}\right]_{x}=\omega\left[\omega_{x x x x}-\frac{\omega_{x x}^{2}}{\omega}\right]
$$

Similarly, the steady-state solution of 14-15 satisfies

$$
\frac{1}{2 \omega_{0}}\left(\omega_{0}^{2} \phi_{0 x}\right)_{x}-\frac{1}{2 \omega_{0}}\left[P\left(\omega_{0}^{2}\right)+\frac{j_{0}^{2}}{\omega_{0}^{2}}\right]_{x x}+\frac{1}{4} \varepsilon^{2} \omega_{0 x x x x}-\frac{1}{4} \varepsilon^{2} \frac{\omega_{0 x x}^{2}}{\omega_{0}}=0 .
$$

Introduce the perturbations of the steady-state

$$
\psi=\omega-\omega_{0}, \quad \eta=j-j_{0}, \quad e=\phi-\phi_{0} .
$$


Then, using $14,(1)-(3)$, and 16 , the evolution equations for $(\psi, \eta, e)$ read as follows:

$$
\begin{gathered}
\eta_{t}+\eta=g_{0}(x, t), \\
\psi_{t t}+\psi_{t}+\frac{1}{4} \varepsilon^{2} \psi_{x x x x}+\frac{1}{2}\left(2 \omega_{0}^{2}+3 \omega_{0} \psi+\phi_{0 x x}+\psi^{2}\right) \psi \\
-\frac{1}{\omega_{0}}\left[\frac{j_{0}}{\omega_{0}^{2}} \eta\right]_{x x}-\left[\left(P^{\prime}\left(\omega_{0}^{2}\right)-\frac{j_{0}^{2}}{\omega_{0}^{4}}\right) \psi_{x}\right]_{x}=g_{1}(x, t)+g_{2}(x, t), \\
e_{x x}=\left(2 \omega_{0}+\psi\right) \psi,
\end{gathered}
$$

with the following initial-boundary values:

$$
\begin{gathered}
\eta(x, 0)=\eta_{0}(x), \quad x \in(0,1), \\
\psi(x, 0)=\psi_{0}(x), \quad \psi_{t}(x, 0)=\theta_{0}(x)=:-\frac{\eta_{0 x}(x)}{2\left(\omega_{0}+\psi_{0}\right)(x)}, \quad x \in(0,1), \\
\psi(0, t)=\psi(1, t)=\psi_{x}(0, t)=\psi_{x}(1, t)=0, \quad t \geq 0, \\
e(0, t)=e(1, t)=0, \quad t \geq 0
\end{gathered}
$$

and the definitions

$$
\begin{aligned}
g_{0}(x, t)= & -\left[\frac{\left(j_{0}+\eta\right)^{2}}{\left(\omega_{0}+\psi\right)^{2}}-\frac{j_{0}^{2}}{\omega_{0}^{2}}+P\left(\left(\omega_{0}+\psi\right)^{2}\right)-P\left(\omega_{0}^{2}\right)\right]_{x} \\
& +\frac{1}{2} \varepsilon^{2}\left(\omega_{0}+\psi\right)^{2}\left(\frac{\left(\omega_{0}+\psi\right)_{x x}}{\omega_{0}+\psi}\right)_{x}-\frac{1}{2} \varepsilon^{2} \omega_{0}^{2}\left(\frac{\omega_{0 x x}}{\omega_{0}}\right)_{x} \\
& +\left(2 \omega_{0}+\psi\right) \psi \phi_{0 x}+\left(\omega_{0}+\psi\right)^{2} e_{x}, \\
g_{1}(x, t)= & \frac{\varepsilon^{2}\left(2 \omega_{0 x x}+\psi_{x x}\right)}{4 \omega_{0}} \psi_{x x}-\frac{\varepsilon^{2}\left(\omega_{0}+\psi\right)_{x x}^{2}}{4\left(\omega_{0}+\psi\right) \omega_{0}} \psi-\frac{\psi_{t}^{2}}{\left(\omega_{0}+\psi\right)} \\
& -\left(\phi_{0 x}+e_{x}\right) \psi_{x}-\omega_{0 x} e_{x}, \\
g_{2}(x, t)= & \frac{1}{2\left(\omega_{0}+\psi\right)}\left[P\left(\left(\omega_{0}+\psi\right)^{2}\right)+\frac{\left(j_{0}+\eta\right)^{2}}{\left(\omega_{0}+\psi\right)^{2}}\right]_{x x}-\frac{1}{2 \omega_{0}}\left[P\left(\omega_{0}^{2}\right)+\frac{j_{0}^{2}}{\omega_{0}^{2}}\right]_{x x} \\
& -\left[\left(P^{\prime}\left(\omega_{0}^{2}\right)-\frac{j_{0}^{2}}{\omega_{0}^{4}}\right) \psi_{x}\right]_{x}-\frac{1}{\omega_{0}}\left[\frac{j_{0}}{\omega_{0}^{2}} \eta\right]_{x x} .
\end{aligned}
$$

Notice that we can write 14 equivalently as

$$
2\left(\omega_{0}+\psi\right) \psi_{t}+\eta_{x}=0
$$

which allows us to estimate the derivatives of $\eta$ in terms of $\psi_{t}$.

2.2. The a-priori estimates. We assume that for given $T>0$, there is a classical solution $(\psi, \eta, e)$ of the IBVP $5-11$ satisfying the regularity condition

$$
(\psi, \eta, e) \in X(T):=C^{0}\left([0, T] ; H^{6}\right) \times C^{0}\left([0, T] ; H^{5}\right) \times C^{0}\left([0, T] ; H^{4}\right) .
$$

We also use the definition

$$
\delta_{T}:=\max _{0 \leq t \leq T}\left(\|\psi(t)\|_{6}+\|\eta(t)\|_{5}\right) .
$$


It is easy to verify that if $\delta_{T}$ is sufficiently small, there are constants $\omega_{-}, \omega_{+}, j_{-}$, and $j_{+}$such that

$$
0<\omega_{-} \leq \omega_{0}+\psi \leq \omega_{+}, \quad j_{-} \leq j_{0}+\eta \leq j_{+}
$$

In the following we assume that $\delta_{T}$ is sufficiently small such that the above estimates hold.

Lemma 2.1. It holds for $(\psi, \eta, e) \in X(T)$ and $(x, t) \in(0,1) \times(0, T)$,

$$
\begin{gathered}
e_{x}(x, t)^{2}+\|e(t)\|_{4}^{2} \leq C\|\psi(t)\|_{2}^{2}, \quad e_{x t}(x, t)^{2}+e_{t}(x, t)^{2}+\left\|e_{t}(t)\right\|_{4}^{2} \leq C\left\|\psi_{t}(t)\right\|_{2}^{2}, \\
\|\eta(t)\|^{2} \leq C\left\|\eta_{0}\right\|^{2} \exp \left\{-c_{0} t\right\}+C\left\|\left(\psi_{t}, \psi, \psi_{x x x}\right)\right\|^{2} \\
\eta(x, t)^{2} \leq C\left\|\eta_{0}\right\|^{2} \exp \left\{-c_{0} t\right\}+C\left\|\left(\psi_{t}, \psi, \psi_{x x x}\right)\right\|^{2} \\
\left\|\eta_{t}(t)\right\|^{2} \leq C\left\|\eta_{0}\right\|^{2} \exp \left\{-c_{0} t\right\}+C\left\|\left(\psi_{t}, \psi, \psi_{x x x}\right)\right\|^{2} \\
\left\|\left(\psi_{x x x x}, \psi_{x x x}\right)\right\|^{2} \leq C\left\|\left(\psi_{t t}, \psi_{t}, \psi, \psi_{x x}, \psi_{x}, \psi_{x t}\right)\right\|^{2}
\end{gathered}
$$

provided that $\delta_{T}+\delta_{0}$ is small enough (see Theorem 1.3 for the definition of $\delta_{0}$ ). Here, $c_{0}, C>0$ are constants independent of time $t$. Here and below the notation $\|(f, g, \ldots)\|^{2}$ means $\|f\|^{2}+\|g\|^{2}+\cdots$.

Proof. The estimates (17) follow directly from the formula

$$
e=\int_{0}^{1} G(x, y)\left(2 \omega_{0}(y)+\psi(y, t)\right) \psi(y, t) d y
$$

and Hölder's inequality. Here, $G(x, y)$ denotes the Green's function

$$
G(x, y)= \begin{cases}x(1-y), & x<y \\ y(1-x), & x>y\end{cases}
$$

To prove (18)-(20), it is sufficient to prove 18. In fact, from (15) follows

$$
\eta^{2} \leq \int_{0}^{1} \eta^{2} d x+2 \int_{0}^{1}\left|\eta_{x} \eta\right| d x \leq C \int_{0}^{1} \eta^{2} d x+C \int_{0}^{1} \psi_{t}^{2} d x
$$

which gives 19 if 18 is proved. In order to see that 20 also follows from 18, we proceed as follows.

We conclude from the boundary condition (10) that there exists $0 \leq x_{1}(t) \leq 1$ such that

$$
\psi_{x}\left(x_{1}(t), t\right)=0
$$

and that there are $x_{2}(t), x_{3}(t)$, and $x_{4}(t)$ satisfying $0 \leq x_{2}(t) \leq x_{1}(t) \leq x_{3}(t) \leq 1$ and $0 \leq x_{2}(t) \leq x_{4}(t) \leq x_{3}(t) \leq 1$ such that

$$
\psi_{x x}\left(x_{2}(t), t\right)=\psi_{x x}\left(x_{3}(t), t\right)=\psi_{x x x}\left(x_{4}(t), t\right)=0 .
$$


Thus, by Poincaré's and Hölder's inequalities, we obtain

$$
\begin{aligned}
\int_{0}^{1} \psi_{x}^{2} d x & \leq C \int_{0}^{1} \psi_{x x}^{2} d x \\
\int_{0}^{1} \psi_{x x}^{2} d x & =\int_{0}^{1}\left(\int_{x_{3}(t)}^{x} \psi_{x x x}(y, t) d y\right)^{2} d x \leq \int_{0}^{1} \psi_{x x x}^{2} d x \\
\int_{0}^{1} \psi_{x x x}^{2} d x & =\int_{0}^{1}\left(\int_{x_{4}(t)}^{x} \psi_{x x x x}(y, t) d y\right)^{2} d x \leq \int_{0}^{1} \psi_{x x x x}^{2} d x
\end{aligned}
$$

Then, using (5), (17), 15, and 23-24, we can estimate

$$
\begin{aligned}
\int_{0}^{1} \eta_{t}^{2} d x \leq & C \int_{0}^{1}\left\{\left(\frac{\left(j_{0}+\eta\right)^{2}}{\left(\omega_{0}+\psi\right)^{2}}-\frac{j_{0}^{2}}{\omega_{0}^{2}}+P\left(\left(\omega_{0}+\psi\right)^{2}\right)-P\left(\omega_{0}^{2}\right)\right)_{x}\right\}^{2} d x \\
& +C \int_{0}^{1}\left[\eta^{2}+\psi^{2} \phi_{0 x}^{2}+e_{x}^{2}\right] d x+C \int_{0}^{1}\left[\left(\left(2 \omega_{0}+\psi\right) \psi\right)_{x x x}^{2}+\left(\left(2 \omega_{0 x}+\psi_{x}\right) \psi_{x}\right)_{x}^{2}\right] d x \\
\leq & C \int_{0}^{1}\left[\eta^{2}+\psi^{2}+\psi_{t}^{2}+\psi_{x}^{2}+\psi_{x x}^{2}+\psi_{x x x}^{2}\right] d x \\
\leq & C \int_{0}^{1}\left[\eta^{2}+\psi^{2}+\psi_{t}^{2}+\psi_{x x x}^{2}\right] d x
\end{aligned}
$$

Hence, estimate 20 follows as soon as 18 is shown.

We now prove 18 . Multiplying (5) by $\eta$, integrating over $x \in(0,1)$ and integrating by parts gives, in view of the boundary conditions (10),

$$
\begin{aligned}
& \frac{1}{2} \frac{d}{d t}\left(\int_{0}^{1} \eta^{2} d x\right)+\int_{0}^{1} \eta^{2} d x \\
\leq & -\left.\left[\eta \frac{\left(j_{0}+\eta\right)^{2}-j_{0}^{2}}{\omega_{0}^{2}}\right]\right|_{0} ^{1}+\int_{0}^{1}\left|\eta\left(\left(2 \omega_{0}+\psi\right) \psi \phi_{0 x}+\left(\omega_{0}+\psi\right)^{2} e_{x}\right)\right| d x \\
& +\int_{0}^{1}\left|\eta_{x}\left(\frac{\left(j_{0}+\eta\right)^{2}}{\left(\omega_{0}+\psi\right)^{2}}-\frac{j_{0}^{2}}{\omega_{0}^{2}}+P\left(\left(\omega_{0}+\psi\right)^{2}\right)-P\left(\omega_{0}^{2}\right)\right)\right| d x \\
& +C \int_{0}^{1}\left(\left|\eta\left(\left(2 \omega_{0}+\psi\right) \psi\right)_{x x x}\right|+\left|\eta_{x} \psi_{x}\left(2 \omega_{0 x}+\psi_{x}\right)\right|\right) d x \\
\triangleq & I_{0}+I_{1}+I_{2}+I_{3} .
\end{aligned}
$$


The integrals $I_{0}, I_{1}, I_{2}$, and $I_{3}$ are estimated as follows.

$$
\begin{aligned}
I_{0} & \leq \int_{0}^{1}\left|\eta_{x} \frac{\left(j_{0}+\eta\right)^{2}-j_{0}^{2}}{\omega_{0}^{2}}+2 \eta \eta_{x} \frac{j_{0}+\eta}{\omega_{0}^{2}}-2 \eta \omega_{0 x} \frac{\left(j_{0}+\eta\right)^{2}-j_{0}^{2}}{\omega_{0}^{3}}\right| d x \\
& \leq\left(C \delta_{0}+\frac{1}{12}\right) \int_{0}^{1} \eta^{2} d x+C \int_{0}^{1} \psi_{t}^{2} d x \\
I_{1} & \leq \frac{1}{12} \int_{0}^{1} \eta^{2} d x+C \int_{0}^{1}\left(\psi^{2}+e_{x}^{2}\right) d x \leq \frac{1}{12} \int_{0}^{1} \eta^{2} d x+C \int_{0}^{1} \psi^{2} d x \\
I_{2} & \leq C \int_{0}^{1}\left|\psi_{t} \eta\right|+\left|\psi \psi_{t}\right| d x \leq \frac{1}{12} \int_{0}^{1} \eta^{2} d x+C \int_{0}^{1}\left[\psi_{t}^{2}+\psi^{2}\right] d x \\
I_{3} & \leq \frac{1}{12} \int_{0}^{1} \eta^{2} d x+C \int_{0}^{1}\left[\psi_{t}^{2}+\psi^{2}+\psi_{x x x}^{2}\right] d x
\end{aligned}
$$

provided that $\delta_{T}+\delta_{0}$ is small enough. In the above estimates we have used 15, 17, 23, and 24. Substituting (27)-(30) into (26) yields

$$
\frac{d}{d t}\left(\int_{0}^{1} \eta^{2} d x\right)+c_{0} \int_{0}^{1} \eta^{2} d x \leq C \int_{0}^{1}\left[\psi_{t}^{2}+\psi^{2}+\psi_{x x x}^{2}\right] d x
$$

where $c_{0} \in\left(0, \frac{4}{3}-C \delta_{0}\right]$ is a constant and $\delta_{0}$ is chosen so small that $C \delta_{0}<\frac{4}{3}$. Applying Gronwall's inequality to 31 gives 18 .

Finally, we prove (21). By (6) and (18), it holds

$$
\begin{aligned}
\int_{0}^{1} \psi_{x x x x}^{2} d x \leq & C \int_{0}^{1}\left(\psi_{t t}^{2}+\psi_{t}^{2}+\psi^{2}+\psi_{x x}^{2}+\psi_{x}^{2}+\psi_{x t}^{2}\right) d x \\
& +C\left(\delta_{T}+\delta_{0}\right) \int_{0}^{1} \psi_{x x x}^{2} d x
\end{aligned}
$$

The combination of (32) and (25) leads to (21), provided that $\delta_{T}+\delta_{0}$ is small enough such that $C\left(\delta_{T}+\delta_{0}\right)<1$.

We prove now uniform estimates in Sobolev spaces for $\psi, \psi_{t}$, and $\psi_{t t}$.

Lemma 2.2. It holds for $(\psi, \eta, e) \in X(T)$ and $0<t<T$,

$$
\begin{gathered}
+\int_{(0,1) \backslash E}\left(P^{\prime}(\mathcal{A})-\frac{j_{0}^{2}}{\mathcal{A}^{2}}\right)\left(\psi_{x}^{2}+\psi_{x t}^{2}\right) d x \\
\qquad C(t)\left\|_{4}^{2}+\right\| \psi_{t}(t)\left\|_{2}^{2}+\right\| \psi_{t t}(t)\left\|^{2}+\right\| e(t) \|_{2}^{2} \\
\leq C\left(\left\|\psi_{0}\right\|_{4}^{2}+\left\|\eta_{0}\right\|_{3}^{2}\right) \exp \left\{-\beta_{3} t\right\},
\end{gathered}
$$

provided that $\delta_{T}+\delta_{0}$ is small enough. Here, $C, \beta_{3}>0$ are constants independent of $t$.

Proof. Step 1: differential inequality for $\psi$ and $\psi_{t}$ in $L^{2}$. We multiply (6) by $\psi$, integrate the resulting equation over $(0,1)$ and integrate by parts, taking into account 
the boundary conditions 10 :

$$
\begin{aligned}
& \frac{d}{d t}\left(\int_{0}^{1}\left[\frac{1}{2} \psi^{2}+\psi \psi_{t}\right] d x\right)-\int_{0}^{1} \psi_{t}^{2} d x+\frac{1}{2} \int_{0}^{1}\left(2 \omega_{0}^{2}+3 \omega_{0} \psi+\phi_{0 x x}+\psi^{2}\right) \psi^{2} d x \\
= & -\int_{0}^{1}\left[\frac{1}{4} \varepsilon^{2} \psi_{x x}^{2}+\left(P^{\prime}\left(\omega_{0}^{2}\right)-\frac{j_{0}^{2}}{\omega_{0}^{4}}\right) \psi_{x}^{2}\right] d x+\int_{0}^{1} \frac{\psi}{\omega_{0}}\left(\frac{j_{0}}{\omega_{0}^{2}} \eta\right)_{x x} d x \\
& +\int_{0}^{1} g_{1} \psi d x+\int_{0}^{1} g_{2} \psi d x \\
\triangleq & I_{4}+I_{5}+I_{6}+I_{7} .
\end{aligned}
$$

We estimate the integrals $I_{4}, \ldots, I_{7}$ term by term. From 23 follows

$$
\begin{aligned}
I_{4}= & -\int_{0}^{1}\left[\frac{1}{4} \varepsilon^{2} \psi_{x x}^{2}+\left(P^{\prime}(\mathcal{A})-\frac{j_{0}^{2}}{\mathcal{A}^{2}}\right) \psi_{x}^{2}\right] d x-\int_{0}^{1}\left(P^{\prime}\left(\omega_{0}\right)-P^{\prime}(\mathcal{A})-\frac{j_{0}^{2}}{\omega_{0}^{4}}+\frac{j_{0}^{2}}{\mathcal{A}^{2}}\right) \psi_{x}^{2} d x \\
\leq & -\frac{1}{4} \varepsilon^{2} \int_{0}^{1} \psi_{x x}^{2} d x-\min _{x \in[0,1]}\left(P^{\prime}(\mathcal{A})-\frac{j_{0}^{2}}{\mathcal{A}^{2}}\right) \int_{E} \psi_{x}^{2} d x-\int_{(0,1) \backslash E}\left(P^{\prime}(\mathcal{A})-\frac{j_{0}^{2}}{\mathcal{A}^{2}}\right) \psi_{x}^{2} d x \\
& -\int_{0}^{1}\left(P^{\prime}\left(\omega_{0}^{2}\right)-P^{\prime}(\mathcal{A})-\frac{j_{0}^{2}}{\omega_{0}^{4}}+\frac{j_{0}^{2}}{\mathcal{A}^{2}}\right) \psi_{x}^{2} d x \\
\leq & -\left(b_{0}+A_{0}\right) \int_{0}^{1} \psi_{x x}^{2} d x-\int_{(0,1) \backslash E}\left(P^{\prime}(\mathcal{A})-\frac{j_{0}^{2}}{\mathcal{A}^{2}}\right) \psi_{x}^{2} d x+C \delta_{0} \int_{0}^{1} \psi_{x x}^{2} d x
\end{aligned}
$$

where $A_{0}$ is given by 26 and

$$
b_{0}=\frac{1}{4}(1-\kappa) \varepsilon^{2}
$$

Note that $A_{0}>0$ by assumption 25 .

Elementary computations, employing 15 and 16 , lead to

$$
\begin{aligned}
\left(\frac{j_{0}}{\omega_{0}^{2}} \eta\right)_{x x}= & -2 \frac{j_{0}}{\omega_{0}^{2}}\left[\left(\omega_{0}+\psi\right) \psi_{x t}+\left(\omega_{0}+\psi\right)_{x} \psi_{t}\right] \\
& -4\left(\frac{j_{0}}{\omega_{0}^{2}}\right)_{x}\left(\omega_{0}+\psi\right) \psi_{t}+\eta\left(\frac{j_{0}}{\omega_{0}^{2}}\right)_{x x} .
\end{aligned}
$$

With this identity, Cauchy's inequality, integration by parts, 18 , and 23, we have

$$
\begin{aligned}
\left|I_{5}\right| \leq & C\left(\delta_{T}+\delta_{0}\right) \int_{0}^{1}\left(\psi^{2}+\psi_{t}^{2}+\eta^{2}\right) d x+\int_{0}^{1}\left|\frac{2 j_{0}}{\omega_{0}^{2}} \psi_{x} \psi_{t}\right| d x \\
\leq & C\left(\delta_{T}+\delta_{0}\right) \int_{0}^{1}\left(\psi_{t}^{2}+\psi^{2}+\psi_{x x x}^{2}\right) d x+a_{0} \int_{0}^{1} \psi_{t}^{2} d x+\frac{1}{4} b_{0} \int_{0}^{1} \psi_{x x}^{2} d x \\
& +C \exp \left\{-c_{0} t\right\} \int_{0}^{1} \eta_{0}^{2} d x,
\end{aligned}
$$

where

$$
a_{0}=4 j_{0}^{2} / \min _{[0,1]} \omega_{0}^{4} b_{0}=\frac{16 j_{0}^{2}}{(1-\kappa) \varepsilon^{2} \min _{[0,1]} \omega_{0}^{4}}
$$

In view of

$$
\left|g_{1}(x, t)\right| \leq C\left(\left|\psi_{x x}\right|+|\psi|+\left|\psi_{t}\right|+\left|\psi_{x}\right|+\left|e_{x}\right|\right)
$$


Cauchy's inequality, (17), and 23, we infer

$$
\left|I_{6}\right| \leq C\left(\delta_{T}+\delta_{0}\right) \int_{0}^{1}\left[\psi_{x x}^{2}+\psi^{2}+\psi_{t}^{2}\right] d x .
$$

By $16,15,(18)$, and (23), we obtain, after a tedious calculation, that

$$
\left|g_{2}(x, t)\right| \leq C\left(\delta_{T}+\delta_{0}\right)\left(\left|\psi_{x x}\right|+\left|\psi_{x}\right|+|\psi|+\left|\psi_{t}\right|+|\eta|\right) .
$$

From the above estimate, 19, and Cauchy's inequality follows

$$
\left|I_{7}\right| \leq C\left(\delta_{T}+\delta_{0}\right) \int_{0}^{1}\left[\psi^{2}+\psi_{x x}^{2}+\psi_{x x x}^{2}+\psi_{t}^{2}\right] d x+C \exp \left\{-c_{0} t\right\} \int_{j_{0}}^{1} \eta_{0}^{2} d x .
$$

Substituting the estimates for $I_{4}, \ldots, I_{7}$ into (35), we conclude

$$
\begin{aligned}
& \frac{d}{d t}\left(\int_{0}^{1}\left[\frac{1}{2} \psi^{2}+\psi_{t} \psi\right] d x\right)-\left(1+a_{0}\right) \int_{0}^{1} \psi_{t}^{2} d x \\
& +\frac{1}{2} \int_{0}^{1}\left(2 \omega_{0}^{2}+3 \omega_{0} \psi+\phi_{0 x x}+\psi^{2}\right) \psi^{2} d x d x \\
& +\left(A_{0}+\frac{3}{4} b_{0}\right) \int_{0}^{1} \psi_{x x}^{2} d x+\int_{(0,1) \backslash E}\left(P^{\prime}(\mathcal{A})-\frac{j_{0}^{2}}{\mathcal{A}^{2}}\right) \psi_{x}^{2} d x \\
& \leq C\left(\delta_{T}+\delta_{0}\right) \int_{0}^{1}\left(\psi^{2}+\psi_{x x}^{2}+\psi_{x x x}^{2}+\psi_{t}^{2}\right) d x+C \exp \left\{-c_{0} t\right\} \int_{0}^{1} \eta_{0}^{2} d x .
\end{aligned}
$$

Multiply now $(6)$ by $\psi_{t}$, integrate the resulting equation over $(0,1)$ and integrate by parts, noticing $\psi_{t}(0, t)=\psi_{t}(1, t)=0$ :

$$
\begin{aligned}
& \quad \frac{1}{2} \frac{d}{d t}\left(\int_{0}^{1}\left[\psi_{t}^{2}+\left(\omega_{0}^{2}+\frac{3}{2} \omega_{0} \psi+\frac{1}{2} \phi_{0 x x}+\frac{1}{4} \psi^{2}\right) \psi^{2}\right] d x\right) \\
& +\frac{1}{2} \frac{d}{d t}\left(\int_{0}^{1}\left[\frac{1}{4} \varepsilon^{2} \psi_{x x}^{2}+\left(P^{\prime}\left(\omega_{0}^{2}\right)-\frac{j_{0}^{2}}{\omega_{0}^{4}}\right) \psi_{x}^{2}\right] d x\right)+\int_{0}^{1} \psi_{t}^{2} d x \\
& =\int_{0}^{1} \omega_{0}^{-1} \psi_{t}\left(\frac{j_{0}}{\omega_{0}^{2}} \eta\right)_{x x} d x+\int_{0}^{1} g_{1} \psi_{t} d x+\int_{0}^{1} g_{2} \psi_{t} d x \\
& \triangleq I_{8}+I_{9}+I_{10} .
\end{aligned}
$$

Employing 37, integration by parts, and (18), we estimate

$$
\begin{aligned}
I_{8} & \leq-\int_{0}^{1} \frac{2 j_{0}}{\omega_{0}^{2}} \psi_{t} \psi_{x t} d x+C\left(\delta_{T}+\delta_{0}\right) \int_{0}^{1}\left(\psi^{2}+\psi_{t}^{2}+\eta^{2}\right) d x \\
& \leq C\left(\delta_{T}+\delta_{0}\right) \int_{0}^{1}\left(\psi_{t}^{2}+\psi^{2}+\psi_{x x x}^{2}\right) d x+C \exp \left\{-c_{0} t\right\} \int_{0}^{1} \eta_{0}^{2} d x .
\end{aligned}
$$

In view of $39,40,17,(18)$, and $(20)$, the integrals $I_{9}$ and $I_{10}$ can be bounded as follows:

$$
\begin{aligned}
\left|I_{9}\right| & \leq C\left(\delta_{T}+\delta_{0}\right) \int_{0}^{1}\left(\psi_{x x}^{2}+\psi^{2}+\psi_{t}^{2}\right) d x \\
\left|I_{10}\right| & \leq C\left(\delta_{T}+\delta_{0}\right) \int_{0}^{1}\left(\psi^{2}+\psi_{x x}^{2}+\psi_{x x x}^{2}+\psi_{t}^{2}\right) d x+C \exp \left\{-c_{0} t\right\} \int_{0}^{1} \eta_{0}^{2} d x .
\end{aligned}
$$


Substitution of the above three estimates into (42) yields

$$
\begin{aligned}
& \quad \frac{1}{2} \frac{d}{d t}\left(\int_{0}^{1}\left[\psi_{t}^{2}+\left(\omega_{0}^{2}+\frac{3}{2} \omega_{0} \psi+\frac{1}{2} \phi_{0 x x}+\frac{1}{4} \psi^{2}\right) \psi^{2}\right] d x\right) \\
& +\frac{1}{2} \frac{d}{d t}\left(\int_{0}^{1}\left[\frac{1}{4} \varepsilon^{2} \psi_{x x}^{2}+\left(P^{\prime}\left(\omega_{0}^{2}\right)-\frac{j_{0}^{2}}{\omega_{0}^{4}}\right) \psi_{x}^{2}\right] d x\right)+\int_{0}^{1} \psi_{t}^{2} d x \\
& \leq C\left(\delta_{T}+\delta_{0}\right) \int_{0}^{1}\left(\psi^{2}+\psi_{x x}^{2}+\psi_{x x x}^{2}+\psi_{t}^{2}\right) d x+C \exp \left\{-c_{0} t\right\} \int_{0}^{1} \eta_{0}^{2} d x .
\end{aligned}
$$

We add 41 and 43 , and multiply by $2\left(1+a_{0}\right)$ (here we recall that $a_{0}$ is denoted by 38), to obtain

$$
\begin{aligned}
& \frac{d}{d t}\left(\int_{0}^{1}\left[\frac{1}{2} \psi^{2}+\psi_{t} \psi+\left(1+a_{0}\right) \psi_{t}^{2}\right] d x\right) \\
& +\frac{d}{d t}\left(\int_{0}^{1}\left(1+a_{0}\right)\left[\omega_{0}^{2}+\frac{3}{2} \omega_{0} \psi+\frac{1}{2} \phi_{0 x x}+\frac{1}{4} \psi^{2}\right] \psi^{2} d x\right) \\
& +\frac{d}{d t}\left(\int_{0}^{1}\left(1+a_{0}\right)\left[\frac{1}{4} \varepsilon^{2} \psi_{x x}^{2}+\left(P^{\prime}\left(\omega_{0}^{2}\right)-\frac{j_{0}^{2}}{\omega_{0}^{4}}\right) \psi_{x}^{2}\right] d x\right) \\
& +\frac{1}{2} \int_{0}^{1}\left[\left(2 \omega_{0}^{2}+3 \omega_{0} \psi+\phi_{0 x x}+\psi^{2}\right) \psi^{2}+2\left(1+a_{0}\right) \psi_{t}^{2}\right] d x \\
& +\left(A_{0}+\frac{3}{4} b_{0}\right) \int_{0}^{1} \psi_{x x}^{2} d x+\int_{(0,1) \backslash E}\left(P^{\prime}(\mathcal{A})-\frac{j_{0}^{2}}{\mathcal{A}^{2}}\right) \psi_{x}^{2} d x \\
& \leq C\left(\delta_{T}+\delta_{0}\right) \int_{0}^{1}\left(\psi^{2}+\psi_{x x}^{2}+\psi_{x x x}^{2}+\psi_{t}^{2}\right) d x+C \exp \left\{-c_{0} t\right\} \int_{0}^{1} \eta_{0}^{2} d x .
\end{aligned}
$$

Applying Gronwall's Lemma to 44, we can estimate the $H^{2}$-norm of $\psi$ and the $L^{2}$ norm of $\psi_{t}$ in terms of the initial data and $\left\|\psi_{x x x}\right\|$. However, the differential inequality for $\psi$ and $\psi_{t}$ is enough for the following considerations.

Step 2: differential inequality for $\psi_{t t}$ in $L^{2}$. The starting point of the following estimates is 6 , differentiated with respect to $t$ :

$$
\begin{aligned}
\psi_{t t t}+\psi_{t t} & +\frac{1}{4} \varepsilon^{2} \psi_{x x x x t}+\left(\omega_{0}^{2}+3 \omega_{0} \psi+\frac{1}{2} \phi_{0 x x}+\frac{3}{2} \psi^{2}\right) \psi_{t} \\
& -\frac{1}{\omega_{0}}\left(\frac{j_{0}}{\omega_{0}^{2}} \eta_{t}\right)_{x x}-\left[\left(P^{\prime}\left(\omega_{0}^{2}\right)-\frac{j_{0}^{2}}{\omega_{0}^{4}}\right) \psi_{x t}\right]_{x}=g_{1 t}(x, t)+g_{2 t}(x, t) .
\end{aligned}
$$

This equation holds pointwise almost everywhere in $(0,1) \times(0, T)$ due to the fact $\psi \in$ $C^{0}\left([0, T] ; H^{6}\right) \cap H^{3}\left(0, T ; L^{2}\right)$ (see the proof of Theorem 1.1). We multiply (45) first by $\psi_{t}$, integrate the resulting equation over $(0,1)$ and integrate by parts, using 7 and the 
boundary conditions $\psi_{t}(0, t)=\psi_{t}(1, t)=\psi_{x t}(0, t)=\psi_{x t}(1, t)=0$ :

$$
\begin{aligned}
& \frac{d}{d t}\left(\int_{0}^{1}\left[\frac{1}{2} \psi_{t}^{2}+\psi_{t} \psi_{t t}\right] d x\right)-\int_{0}^{1} \psi_{t t}^{2} d x+\int_{0}^{1}\left[\omega_{0}^{2}+3 \omega_{0} \psi+\frac{1}{2} \phi_{0 x x}+\frac{3}{2} \psi^{2}\right] \psi_{t}^{2} d x \\
= & -\int_{0}^{1}\left[\frac{1}{4} \varepsilon^{2} \psi_{x x t}^{2}+\left(P^{\prime}\left(\omega_{0}^{2}\right)-\frac{j_{0}^{2}}{\omega_{0}^{4}}\right) \psi_{x t}^{2}\right] d x+\int_{0}^{1} \frac{1}{\omega_{0}} \psi_{t}\left(\frac{j_{0}}{\omega_{0}^{2}} \eta_{t}\right)_{x x} d x \\
& +\int_{0}^{1} g_{1 t} \psi_{t} d x+\int_{0}^{1} g_{2 t} \psi_{t} d x \\
\triangleq & I_{12}+I_{13}+I_{14}+I_{15} .
\end{aligned}
$$

Applying an argument similar to 36 , it follows

$$
I_{12} \leq-\left(A_{0}+b_{0}\right) \int_{0}^{1} \psi_{x x t}^{2} d x-\int_{(0,1) \backslash E}\left(P^{\prime}(\mathcal{A})-\frac{j_{0}^{2}}{\mathcal{A}^{2}}\right) \psi_{x t}^{2} d x+C \delta_{0} \int_{0}^{1} \psi_{x x t}^{2} d x
$$

where we have used

$$
\int_{E} \psi_{x t}^{2} d x \leq \int_{0}^{1} \psi_{x t}^{2} d x \leq \int_{0}^{1} \psi_{x x t}^{2} d x
$$

based on the facts $\psi_{x t}(0, t)=\psi_{x t}(1, t)=0$. By (15), (20), and (47), we have, after integration by parts,

$$
\begin{aligned}
I_{13}= & -2 \int_{0}^{1} \frac{j_{0}}{\omega_{0}^{3}} \psi_{t}\left(\left(\psi+\omega_{0}\right) \psi_{x t t}+2 \psi_{t} \psi_{x t}+\left(\omega_{0}+\psi\right)_{x} \psi_{t t}\right) d x \\
& -4 \int_{0}^{1} \omega_{0}^{-1}\left(\frac{j_{0}}{\omega_{0}^{2}}\right)_{x} \psi_{t}\left(\psi_{t}^{2}+\left(\omega_{0}+\psi\right) \psi_{t t}\right) d x+\int_{0}^{1} \omega_{0}^{-1}\left(\frac{j_{0}}{\omega_{0}^{2}}\right)_{x x} \psi_{t} \eta_{t} d x \\
\leq & C\left(\delta_{T}+\delta_{0}\right) \int_{0}^{1}\left(\psi_{t t}^{2}+\psi_{x t}^{2}+\psi_{t}^{2}+\eta_{t}^{2}\right) d x \\
& +2 \int_{0}^{1}\left|\left(\frac{j_{0}\left(\omega_{0}+\psi\right)}{\omega_{0}^{3}}\right)_{x} \psi_{t} \psi_{t t}\right| d x+2 \int_{0}^{1}\left|\frac{j_{0}}{\omega_{0}^{3}}\left(\omega_{0}+\psi\right) \psi_{x t} \psi_{t t}\right| d x \\
\leq & C\left(\delta_{T}+\delta_{0}\right) \int_{0}^{1}\left(\psi_{t t}^{2}+\psi_{t}^{2}+\psi_{x x x}^{2}+\psi_{x x t}^{2}\right) d x \\
& +C \exp \left\{-c_{0} t\right\} \int_{0}^{1} \eta_{0}^{2} d x+a_{0} \int_{0}^{1} \psi_{t t}^{2} d x+\frac{1}{4} b_{0} \int_{0}^{1} \psi_{x x t}^{2} d x .
\end{aligned}
$$

Elementary computations yield the estimate

$$
\left|g_{1 t}(x, t)\right| \leq C\left(\delta_{T}+\delta_{0}\right)\left(\left|\psi_{x x t}\right|+\left|\psi_{t t}\right|+\left|\psi_{t}\right|+\left|\psi_{x t}\right|+\left|e_{x t}\right|\right),
$$

which implies, in view of Cauchy's inequality, (21), and (47), that

$$
\left|I_{14}\right| \leq C\left(\delta_{T}+\delta_{0}\right) \int_{0}^{1}\left(\psi_{x x t}^{2}+\psi_{t}^{2}+\psi_{t t}^{2}\right) d x
$$


After a tedious computation, it follows from 16 that

$$
\begin{aligned}
\left|g_{2 t}(x, t)\right| \leq & C\left(\delta_{T}+\delta_{0}\right)\left(\left|\psi_{x x t}\right|+\left|\psi_{t t}\right|+\left|\psi_{x t}\right|+\left|\psi_{t}\right|+\left|\eta_{t}\right|\right) \\
& +\left(\frac{j_{0}+\eta}{\left(\omega_{0}+\psi\right)^{3}}-\frac{j_{0}}{\omega_{0}^{3}}\right) \eta_{x x t} \\
\leq & C\left(\delta_{T}+\delta_{0}\right)\left(\left|\psi_{x x t}\right|+\left|\psi_{x x}\right|+\left|\psi_{t t}\right|+\left|\psi_{x t}\right|+\left|\psi_{t}\right|+\left|\eta_{t}\right|\right) \\
& -2\left(\omega_{0}+\psi\right)\left(\frac{j_{0}+\eta}{\left(\omega_{0}+\psi\right)^{3}}-\frac{j_{0}}{\omega_{0}^{3}}\right) \psi_{x t t},
\end{aligned}
$$

where we have used the equation

$$
4 \psi_{x x} \psi_{t}+2\left(\omega_{0}+\psi\right) \psi_{x t t}+2\left(\omega_{0}+\psi\right)_{x} \psi_{t t}+\eta_{x x t}=0
$$

Using $49,(18), 47,(24)$, and the fact $\psi_{t}(0, t)=\psi_{t}(1, t)=0$, we can estimate $I_{15}$, after integration by parts, as follows:

$$
\begin{aligned}
I_{15} \leq & \left.\delta_{T}+\delta_{0}\right) \int_{0}^{1}\left(\psi_{x x t}^{2}+\psi_{t t}^{2}+\psi_{t}^{2}+\psi^{2}+\psi_{x x}^{2}+\psi_{x x x}^{2}\right) d x \\
& +2 \int_{0}^{1}\left(\omega_{0}+\psi\right)\left(\frac{j_{0}+\eta}{\left(\omega_{0}+\psi\right)^{3}}-\frac{j_{0}}{\omega_{0}^{3}}\right) \psi_{x t} \psi_{t t} d x \\
& +2 \int_{0}^{1}\left(\left(\omega_{0}+\psi\right)\left[\frac{j_{0}+\eta}{\left(\omega_{0}+\psi\right)^{3}}-\frac{j_{0}}{\omega_{0}^{3}}\right]\right)_{x} \psi_{t} \psi_{t t} d x \\
\leq & C\left(\delta_{T}+\delta_{0}\right) \int_{0}^{1}\left(\psi_{x x t}^{2}+\psi_{t t}^{2}+\psi_{t}^{2}+\psi^{2}+\psi_{x x}^{2}+\psi_{x x x}^{2}\right) d x
\end{aligned}
$$

Substituting the above estimates for $I_{12}, \ldots, I_{15}$ into (46), we conclude

$$
\begin{aligned}
& \quad \frac{d}{d t}\left(\int_{0}^{1}\left[\frac{1}{2} \psi_{t}^{2}+\psi_{t} \psi_{t t}\right] d x\right)-\left(1+a_{0}\right) \int_{0}^{1} \psi_{t t}^{2} d x \\
& +\int_{0}^{1}\left[\omega_{0}^{2}+3 \omega_{0} \psi+\frac{1}{2} \phi_{0 x x}+\frac{3}{2} \psi^{2}\right] \psi_{t}^{2} d x \\
& +\left(A_{0}+\frac{3}{4} b_{0}\right) \int_{0}^{1} \psi_{x x t}^{2} d x+\int_{(0,1) \backslash E}\left(P^{\prime}(\mathcal{A})-\frac{j_{0}^{2}}{\mathcal{A}^{2}}\right) \psi_{x t}^{2} d x \\
& \leq C\left(\delta_{T}+\delta_{0}\right) \int_{0}^{1}\left(\psi_{t}^{2}+\psi^{2}+\psi_{t t}^{2}+\psi_{x x}^{2}+\psi_{x x t}^{2}+\psi_{x x x}^{2}\right) d x \\
& \quad+C \exp \left\{-c_{0} t\right\} \int_{0}^{1} \eta_{0}^{2} d x .
\end{aligned}
$$


The next step is to multiply (45) by $\psi_{t t}$, to integrate the resulting equation over $(0,1)$ and to integrate by parts, using $\psi_{t t}(0, t)=\psi_{t t}(1, t)=0$, which yields

$$
\begin{aligned}
& \frac{1}{2} \frac{d}{d t}\left(\int_{0}^{1}\left[\psi_{t t}^{2}+\left(\omega_{0}^{2}+3 \omega_{0} \psi+\frac{1}{2} \phi_{0 x x}+\frac{3}{2} \psi^{2}\right) \psi_{t}^{2}\right] d x\right) \\
& +\frac{1}{2} \frac{d}{d t}\left(\int_{0}^{1}\left[\frac{1}{4} \varepsilon^{2} \psi_{x x t}^{2}+\left(P^{\prime}\left(\omega_{0}^{2}\right)-\frac{j_{0}^{2}}{\omega_{0}^{4}}\right) \psi_{x t}^{2}\right] d x\right) \\
& -\frac{3}{2} \int_{0}^{1}\left(\omega_{0}+\psi\right) \psi_{t}^{3} d x+\int_{0}^{1} \psi_{t t}^{2} d x \\
& =\int_{0}^{1} \omega_{0}^{-1} \psi_{t t}\left(\frac{j_{0}}{\omega_{0}^{2}} \eta_{t}\right)_{x x} d x+\int_{0}^{1} g_{1 t} \psi_{t t} d x+\int_{0}^{1} g_{2 t} \psi_{t t} d x \\
& \triangleq I_{16}+I_{17}+I_{18} .
\end{aligned}
$$

By $15,50,(20),(23),(47)$, and integration by parts, we have

$$
\begin{aligned}
I_{16}= & -2 \int_{0}^{1} \frac{j_{0}}{\omega_{0}^{3}}\left(\omega_{0}+\psi\right) \psi_{t t} \psi_{x t t} d x-2 \int_{0}^{1} \frac{j_{0}}{\omega_{0}^{3}}\left(2 \psi_{t} \psi_{x t}+\left(\omega_{0}+\psi\right)_{x} \psi_{t t}\right) d x \\
& -4 \int_{0}^{1} \frac{1}{\omega_{0}}\left(\frac{j_{0}}{\omega_{0}^{2}}\right)_{x} \psi_{t t}\left(\psi_{t}^{2}+\left(\omega_{0}+\psi\right) \psi_{t t}\right) d x+\int_{0}^{1} \frac{1}{\omega_{0}}\left(\frac{j_{0}}{\omega_{0}^{2}}\right)_{x x} \psi_{t t} \eta_{t} d x \\
\leq & C\left(\delta_{T}+\delta_{0}\right) \int_{0}^{1}\left(\psi_{t t}^{2}+\psi_{t}^{2}+\psi^{2}+\psi_{x x t}^{2}+\psi_{x x x}^{2}\right) d x+C \exp \left\{-c_{0} t\right\} \int_{0}^{1} \eta_{0}^{2} d x
\end{aligned}
$$

From (48), (17), and (47), it follows

$$
\left|I_{17}\right| \leq C\left(\delta_{T}+\delta_{0}\right) \int_{0}^{1}\left[\psi_{x x t}^{2}+\psi_{t}^{2}+\psi_{t t}^{2}\right] d x
$$

Finally, in view of (49), (18), (20), and integration by parts, it holds

$$
\begin{aligned}
I_{18} \leq & \left(\delta_{T}+\delta_{0}\right) \int_{0}^{1}\left(\psi_{x x t}^{2}+\psi_{t t}^{2}+\psi_{t}^{2}+\psi^{2}+\psi_{x x x}^{2}\right) d x \\
& -2 \int_{0}^{1}\left(\omega_{0}+\psi\right)\left(\frac{j_{0}+\eta}{\left(\omega_{0}+\psi\right)^{3}}-\frac{j_{0}}{\omega_{0}^{3}}\right) \psi_{t t} \psi_{x t t} d x \\
\leq & \left(\delta_{T}+\delta_{0}\right) \int_{0}^{1}\left[\psi_{x x t}^{2}+\psi_{t t}^{2}+\psi_{t}^{2}+\psi^{2}+\psi_{x x x}^{2}\right] d x
\end{aligned}
$$


Substituting the estimates for the integrals $I_{16}, I_{17}$, and $I_{18}$ into (52) gives

$$
\begin{aligned}
& \quad \frac{1}{2} \frac{d}{d t}\left(\int_{0}^{1}\left[\psi_{t t}^{2}+\left(\omega_{0}^{2}+3 \omega_{0} \psi+\frac{1}{2} \phi_{0 x x}+\frac{3}{2} \psi^{2}\right) \psi_{t}^{2}\right] d x\right) \\
& +\frac{1}{2} \frac{d}{d t}\left(\int_{0}^{1}\left[\frac{1}{4} \varepsilon^{2} \psi_{x x t}^{2}+\left(P^{\prime}\left(\omega_{0}^{2}\right)-\frac{j_{0}^{2}}{\omega_{0}^{4}}\right) \psi_{x t}^{2}\right] d x\right) \\
& \quad-\frac{3}{2} \int_{0}^{1}\left(\omega_{0}+\psi\right) \psi_{t}^{3} d x+\int_{0}^{1} \psi_{t t}^{2} d x \\
& \leq C\left(\delta_{T}+\delta_{0}\right) \int_{0}^{1}\left(\psi_{x x t}^{2}+\psi_{t t}^{2}+\psi_{t}^{2}+\psi^{2}+\psi_{x x}^{2}+\psi_{x x x}^{2}\right) d x \\
& \quad+C \exp \left\{-c_{0} t\right\} \int_{0}^{1} \eta_{0}^{2} d x
\end{aligned}
$$

Now we add inequalities 51 and 53 , and multiply by $2\left(1+a_{0}\right)$, to infer

$$
\begin{aligned}
& \frac{d}{d t}\left(\int_{0}^{1}\left[\frac{1}{2} \psi_{t}^{2}+\psi_{t} \psi_{t t}+\left(1+a_{0}\right) \psi_{t t}^{2}\right] d x\right) \\
& +\left(1+a_{0}\right) \frac{d}{d t}\left(\int_{0}^{1}\left[\omega_{0}^{2}+3 \omega_{0} \psi+\frac{1}{2} \phi_{0 x x}+\frac{3}{2} \psi^{2}\right] \psi_{t}^{2} d x\right) \\
& +\left(1+a_{0}\right) \frac{d}{d t}\left(\int_{0}^{1}\left[\frac{1}{4} \varepsilon^{2} \psi_{x x t}^{2}+\left(P^{\prime}\left(\omega_{0}^{2}\right)-\frac{j_{0}^{2}}{\omega_{0}^{4}}\right) \psi_{x t}^{2}\right] d x\right) \\
& +\left(1+a_{0}\right) \int_{0}^{1} \psi_{t t}^{2} d x+\int_{0}^{1}\left[\omega_{0}^{2}+3 \omega_{0} \psi+\frac{1}{2} \phi_{0 x x}+\frac{3}{2} \psi^{2}\right] \psi_{t}^{2} d x \\
& +\left(A_{0}+\frac{3}{4} b_{0}\right) \int_{0}^{1} \psi_{x x t}^{2} d x+\int_{(0,1) \backslash E}\left(P^{\prime}(\mathcal{A})-\frac{j_{0}^{2}}{\mathcal{A}^{2}}\right) \psi_{x t}^{2} d x \\
& \leq C\left(\delta_{T}+\delta_{0}\right) \int_{0}^{1}\left(\psi_{t}^{2}+\psi_{t t}^{2}+\psi_{x x}^{2}+\psi_{x x t}^{2}+\psi_{x x x}^{2}+\psi^{2}\right) d x \\
& +\exp \left\{-c_{0} t\right\} \cdot \int_{0}^{1} \eta_{0}^{2} d x
\end{aligned}
$$


Step 3: combination of the estimates for $\psi, \psi_{t}$, and $\psi_{t t}$. We combine the estimates (44) and (54) and obtain for some constant $\beta_{1}>0$, using (21),

$$
\begin{aligned}
& \frac{d}{d t}\left(\int_{0}^{1}\left[\frac{1}{2}\left(\psi^{2}+\psi_{t}^{2}\right)+\psi_{t}\left(\psi+\psi_{t t}\right)+\left(1+a_{0}\right)\left(\psi_{t}^{2}+\psi_{t t}^{2}\right)\right] d x\right) \\
& +\left(1+a_{0}\right) \frac{d}{d t}\left(\int_{0}^{1}\left[\omega_{0}^{2}+\frac{3}{2} \omega_{0} \psi+\frac{1}{2} \phi_{0 x x}+\frac{1}{4} \psi^{2}\right] \psi^{2} d x\right) \\
& +\left(1+a_{0}\right) \frac{d}{d t}\left(\int_{0}^{1}\left[\omega_{0}^{2}+3 \omega_{0} \psi+\frac{1}{2} \phi_{0 x x}+\frac{3}{2} \psi^{2}\right] \psi_{t}^{2} d x\right) \\
& +\left(1+a_{0}\right) \frac{d}{d t}\left(\int_{0}^{1}\left[\frac{1}{4} \varepsilon^{2}\left(\psi_{x x}^{2}+\psi_{x x t}^{2}\right)+\left(P^{\prime}\left(\omega_{0}^{2}\right)-\frac{j_{0}^{2}}{\omega_{0}^{4}}\right)\left(\psi_{x}^{2}+\psi_{x t}^{2}\right)\right] d x\right) \\
& +\beta_{1} \int_{0}^{1}\left[\psi^{2}+\psi_{t}^{2}+\psi_{t}^{2}+\psi_{t t}^{2}+\psi_{x x t}^{2}+\psi_{x x}^{2}\right] d x \\
& +\beta_{1} \int_{(0,1) \backslash E}\left(P^{\prime}(\mathcal{A})-\frac{j_{0}^{2}}{\mathcal{A}^{2}}\right)\left(\psi_{x}^{2}+\psi_{x t}^{2}\right) d x \\
& \leq C \exp \left\{-c_{0} t\right\} \int_{0}^{1} \eta_{0}^{2} d x,
\end{aligned}
$$

provided that $\delta_{T}+\delta_{0}$ is small enough.

There exist constants $\beta_{2}, \beta_{3}>0$ such that

$$
\begin{aligned}
& \beta_{2} \int_{0}^{1}\left[\psi^{2}+\psi_{t}^{2}+\psi_{x t}^{2}+\psi_{t t}^{2}+\psi_{x x t}^{2}+\psi_{x x}^{2}\right] d x \\
& +\beta_{2} \int_{(0,1) \backslash E}\left(P^{\prime}(\mathcal{A})-\frac{j_{0}^{2}}{\mathcal{A}^{2}}\right)\left(\psi_{x}^{2}+\psi_{x t}^{2}\right) d x \\
& \leq \int_{0}^{1}\left[\frac{1}{2}\left(\psi^{2}+\psi_{t}^{2}\right)+\psi_{t}\left(\psi+\psi_{t t}\right)+\left(1+a_{0}\right)\left(\psi_{t}^{2}+\psi_{t t}^{2}\right)\right] d x \\
& \quad+\left(1+a_{0}\right) \int_{0}^{1}\left[\omega_{0}^{2}+\frac{3}{2} \omega_{0} \psi+\frac{1}{2} \phi_{0 x x}+\frac{1}{4} \psi^{2}\right] \psi^{2} d x \\
& \quad+\left(1+a_{0}\right) \int_{0}^{1}\left[\omega_{0}^{2}+3 \omega_{0} \psi+\frac{1}{2} \phi_{0 x x}+\frac{3}{2} \psi^{2}\right] \psi_{t}^{2} d x \\
& \quad+\left(1+a_{0}\right) \int_{0}^{1}\left[\frac{1}{4} \varepsilon^{2}\left(\psi_{x x}^{2}+\psi_{x x t}^{2}\right)+\left(P^{\prime}\left(\omega_{0}^{2}\right)-\frac{j_{0}^{2}}{\omega_{0}^{4}}\right)\left(\psi_{x}^{2}+\psi_{x t}^{2}\right)\right] d x \\
& \leq \beta_{3}^{-1} \beta_{1} \int_{0}^{1}\left[\psi^{2}+\psi_{t}^{2}+\psi_{x t}^{2}+\psi_{t t}^{2}+\psi_{x x t}^{2}+\psi_{x x}^{2}\right] d x \\
& \quad+\beta_{3}^{-1} \beta_{1} \int_{(0,1) \backslash E}\left(P^{\prime}(\mathcal{A})-\frac{j_{0}^{2}}{\mathcal{A}^{2}}\right)\left(\psi_{x}^{2}+\psi_{x t}^{2}\right) d x .
\end{aligned}
$$

Thus, applying Gronwall's inequality to 55 , we finally obtain

$$
\begin{aligned}
& \int_{0}^{1}\left[\psi^{2}+\psi_{t}^{2}+\psi_{x t}^{2}+\psi_{t t}^{2}+\psi_{x x t}^{2}+\psi_{x x}^{2}\right] d x+\int_{(0,1) \backslash E}\left(P^{\prime}(\mathcal{A})-\frac{j_{0}^{2}}{\mathcal{A}^{2}}\right)\left(\psi_{x}^{2}+\psi_{x t}^{2}\right) d x \\
\leq & C\left(\left\|\psi_{0}\right\|_{4}^{2}+\left\|\eta_{0}\right\|_{3}^{2}\right) \exp \left\{-\beta_{3} t\right\},
\end{aligned}
$$


provided that $\delta_{T}+\delta_{0}$ is small enough.

The combination of (56), (17), (21), and 7 gives assertion 34 . Thus, the lemma is proved.

We also obtain bounds for higher-order estimates for $\psi$.

Lemma 2.3. It holds for $(\psi, \eta, e) \in X(T)$ and $0<t<T$

$$
\begin{aligned}
& \left\|\partial_{x}^{4} \psi(t)\right\|_{2}^{2}+\left\|\psi_{x t t}(t)\right\|_{1}^{2}+\left\|\psi_{t t t}(t)\right\|^{2}+\|e(t)\|_{4}^{2}+\int_{(0,1) \backslash E}\left(P^{\prime}(\mathcal{A})-\frac{j_{0}^{2}}{\mathcal{A}^{2}}\right) \psi_{x t t}^{2} d x \\
\leq & C\left(\|\left(\psi_{0}\left\|_{6}^{2}+\right\| \eta_{0} \|_{5}^{2}\right) \exp \left\{-\beta_{4} t\right\},\right.
\end{aligned}
$$

provided that $\delta_{T}+\delta_{0}$ is small enough. Here, $C, \beta_{4}>0$ are constants independent of $t$.

Proof. For the proof of the lemma take the time derivative of 45 and estimate similarly as in Lemmas 2.1 and 2.2. As the estimates are analogous to those of the proofs of Lemmas 2.1-2.2, we omit the details.

Proof of Theorem 1.5. By Theorem 1.1, there exists a solution $(\omega, j, \phi)$ of the IBVP 14 19 for $t \in\left[0, T_{*}\right]$. With the help of Lemmas 2.1-2.3, we infer that the local solution $(\omega, j, \phi)$ of the IBVP $14-19$ satisfies, for $t \in\left[0, T_{*}\right]$,

$$
\left\|\left(\omega-\omega_{0}, j-j_{0}, \phi-\phi_{0}\right)(t)\right\|_{H^{6} \times H^{5} \times H^{4}}^{2} \leq C\left(\left\|\psi_{0}\right\|_{6}^{2}+\left\|\eta_{0}\right\|_{5}^{2}\right) \exp \left\{-\Lambda_{0} t\right\},
$$

where $C, \Lambda_{0}>0$ are constants independent of $t$. Choosing the initial data $\left\|\psi_{0}\right\|_{6}+\left\|\eta_{0}\right\|_{5}$ so small that

$$
C\left(\left\|\psi_{0}\right\|_{6}^{2}+\left\|\eta_{0}\right\|_{5}^{2}\right)<\delta_{T_{*}}
$$

we conclude first, by the Sobolev embedding theorem and 58, that $w>0$ in $[0,1] \times\left[0, T_{*}\right]$, and second, by the usual continuity argument, that $(\omega, j, \phi)$ exists globally in time and satisfies 29 .

3. Proof of Theorem 1.1. The idea of the proof of Theorem 1.1 is to linearize Eqs. 14-16 around the initial state and to construct a sequence of approximate solutions of the linearized problem converging to a solution of the original problem. First we need to study the regularity properties of a certain semilinear fourth-order wave equation.

3.1. A semilinear fourth-order wave equation. Consider the two Hilbert spaces $H_{0}^{2}$ and $L^{2}$ on $(0,1)$, endowed with the scalar products $\langle\cdot, \cdot\rangle$ and $(\cdot, \cdot)$ and corresponding norms $|\cdot|_{H_{0}^{2}}=|\cdot|_{2}$ and $\|\cdot\|$, respectively. Furthermore, we consider the following initial-value problem on $L^{2}$ :

$$
\begin{aligned}
& u^{\prime \prime}+u^{\prime}+\nu A u+u+\mathcal{L} u^{\prime}=F(t), \quad t>0, \\
& u(0)=u_{0}, . u^{\prime}(0)=u_{1},
\end{aligned}
$$

where the primes denote derivatives with respect to time, $\tau, \nu>0$ are constants, $A=\partial_{x}^{4}$ is an operator defined on

$$
D(A)=H_{0}^{2} \cap H^{4}=\left\{u \in H^{4} ;\left.u\right|_{x=0,1}=\left.u_{x}\right|_{x=0,1}=0\right\},
$$


and the operators $\mathcal{L}$ and $F$ are given by

$$
\begin{aligned}
\langle\mathcal{L} u, v\rangle & =\int_{0}^{1} b(x, t) u_{x} v d x, \quad u, v \in H_{0}^{2}, \\
(F(t), v) & =\int_{0}^{1} f(x, t) v d x, \quad v \in L^{2},
\end{aligned}
$$

where $b, f:[0,1] \times[0, T] \rightarrow \mathbb{R}$ are measurable functions.

Related to the operator $A$, we introduce the coercive, continuous, symmetric bilinear form $a(u, v)$

$$
a(u, v)=\nu \int_{0}^{1} u_{x x} v_{x x} d x \quad \forall u, v \in H_{0}^{2} .
$$

There exist a complete orthonormal family of eigenvectors $\left\{r_{i}\right\}_{i \in \mathbb{N}}$ of $L^{2}$ and a family of eigenvalues $\left\{\mu_{i}\right\}_{i \in \mathbb{N}}$ such that $0<\mu_{1} \leq \mu_{2} \leq \cdots$ and $\mu_{i} \rightarrow \infty$ as $i \rightarrow \infty$.

The family $\left\{r_{i}\right\}_{i \in N}$ is also orthogonal for $a(u, v)$ on $H_{0}^{2}$, i.e.,

$$
\left(r_{i}, r_{j}\right)=\delta_{i j}, \quad a\left(r_{i}, r_{j}\right)=\nu\left\langle A r_{i}, r_{j}\right\rangle=\nu \delta_{i j} \quad \forall i, j .
$$

Using the Faedo-Galerkin method [26, 28], it is possible to prove the existence of solutions of $(59)-(60)$. The result is summarized in the following theorem.

THEOREM 3.1. Let $T_{0}>0$ and assume that

$$
F \in H^{1}\left(0, T_{0} ; L^{2}\right), \quad b \in C^{1}\left(\left[0, T_{0}\right] ; H^{2}\right) \cap W^{2, \infty}\left(0, T_{0} ; H^{1}\right) .
$$

Then, if $u_{0} \in H^{4} \cap H_{0}^{2}$ and $u_{1} \in H_{0}^{2}$, there exists a solution of (59)-(60) satisfying

$$
u \in C\left(\left[0, T_{0}\right] ; H^{4} \cap H_{0}^{2}\right) \cap C^{1}\left(\left[0, T_{0}\right] ; H_{0}^{2}\right) \cap C^{2}\left(\left[0, T_{0}\right] ; L^{2}\right) .
$$

Moreover, assume additionally that

$$
F \in H^{2}\left(0, T_{0} ; L^{2}\right) \cap C\left(\left[0, T_{0}\right] ; H^{2}\right) .
$$

Then, if $u_{0} \in H^{6} \cap H_{0}^{2}$ and $u_{1} \in H^{4} \cap H_{0}^{2}$ satisfy $\nu A u_{0}+\mathcal{L}\left(u_{1}\right)-F(0) \in H_{0}^{2}$, it holds

$$
u \in C^{i}\left(\left[0, T_{0}\right] ; H^{6-2 i} \cap H_{0}^{2}\right) \cap C^{3}\left(\left[0, T_{0}\right] ; L^{2}\right), \quad i=0,1,2 .
$$

Proof. The existence of solutions of 59-60 and the regularity property 63 can be shown by applying the Faedo-Galerkin method as in [19]. The regularity property 64 follows from 63 by considering the problem for the new variable $v=u^{\prime}$. As the proof is standard, we omit the details.

3.2. Local existence. In this section we prove Theorem 1.1. For simplicity, we set $\tau=1$. We linearize Eqs. $14-16$ around the initial state $\left(\omega_{1}, j_{1}, \phi_{1}\right)$ where $\phi_{1}$ solves the Poisson equation 16 and 19 with $\omega$ replaced by $\omega_{1}$, and prove the local-in-time existence for the perturbation $(\psi, \eta, e)=\left(\omega-\omega_{1}, j-j_{1}, \phi-\phi_{1}\right)$. For this, we reformulate the original initial-boundary value problem 14-19. It is sufficient to carry out the reformulation for Eqs. 14,16 , and 1 because of 15 . For given $U^{p}=\left(\psi_{p}, \eta_{p}, e_{p}\right)$ we obtain the following linearized problems for $U^{p+1}=\left(\psi_{p+1}, \eta_{p+1}, e_{p+1}\right), p \in \mathbb{N}$, writing " $\partial_{x}$ " for the spatial 
derivative and " $"$ " for the time derivative:

$$
\begin{aligned}
& \left\{\begin{array}{l}
\eta_{p+1}^{\prime}+\eta_{p+1}=g_{3}\left(x, U^{p}\right) \\
\eta_{p+1}(x, 0)=0
\end{array}\right. \\
& \left\{\begin{array}{l}
\psi_{p+1}^{\prime \prime}+\psi_{p+1}^{\prime}+\nu \partial_{x}^{4} \psi_{p+1}+\psi_{p+1}+k\left(x, U^{p}\right) \partial_{x} \psi_{p+1}^{\prime}=g_{4}\left(x, U^{p}\right) \\
\psi_{p+1}(x, 0)=0, \quad \psi_{p+1}^{\prime}(x, 0)=\theta_{1}(x):=-\frac{\partial_{x} j_{1}}{2 \omega_{1}} \\
\psi_{p+1}(0, t)=\psi_{p+1}(1, t)=\partial_{x} \psi_{p+1}(0, t)=\partial_{x} \psi_{p+1}(1, t)=0
\end{array}\right. \\
& \left\{\begin{array}{l}
\partial_{x}^{2} e_{p+1}=\left(2 \omega_{1}+\psi_{p}\right) \psi_{p} \\
e_{p+1}(0, t)=e_{p+1}(1, t)=0
\end{array}\right.
\end{aligned}
$$

where $\nu=\frac{1}{4} \varepsilon^{2}$ and

$$
\begin{aligned}
g_{3}\left(x, U^{p}\right)= & \frac{4\left(j_{1}+\eta_{p}\right)}{\omega_{1}+\psi_{p}} \psi_{p}^{\prime}-\left(j_{1}+\eta_{p}\right)^{2}\left[\frac{1}{\left(\omega_{1}+\psi_{p}\right)^{2}}\right]_{x}-P\left(\left(\omega_{1}+\psi_{p}\right)^{2}\right)_{x}-j_{1} \\
& +\left(w_{1}+\psi_{p}\right)^{2}\left(\phi_{1}+e_{p}\right)_{x}+\frac{1}{2} \varepsilon^{2}\left(w_{1}+\psi_{p}\right)^{2}\left[\frac{\left(w_{1}+\psi_{p}\right)_{x x}}{w_{1}+\psi_{p}}\right]_{x}, \\
k\left(x, U^{p}\right)= & \frac{2\left(j_{1}+\eta_{p}\right)}{\left(\omega_{1}+\psi_{p}\right)^{2}}, \\
g_{4}\left(x, U^{p}\right)= & -\frac{1}{2\left(\omega_{1}+\psi_{p}\right)}\left(\left(\omega_{1}+\psi_{p}\right)^{2}\left(\phi_{1}+e_{p}\right)_{x}\right)_{x}+\frac{1}{2\left(\omega_{1}+\psi_{p}\right)} P\left(\left(\omega_{1}+\psi_{p}\right)^{2}\right)_{x x} \\
& -\frac{1}{4} \varepsilon^{2} \omega_{1 x x x x}+\frac{1}{4} \varepsilon^{2} \frac{\left(\omega_{1}+\psi_{p}\right)_{x x}^{2}}{\omega_{1}+\psi_{p}}-\psi_{p}+\frac{3\left(\psi_{p}^{\prime}\right)^{2}}{\omega_{1}+\psi_{p}} \\
& +\frac{\left(j_{1}+\eta_{p}\right)^{2}}{2\left(\omega_{1}+\psi_{p}\right)}\left[\frac{1}{\left(\omega_{1}+\psi_{p}\right)^{2}}\right]_{x x}-3\left[\frac{1}{\left(\omega_{1}+\psi_{p}\right)^{2}}\right]_{x}\left(j_{1}+\eta_{p}\right) \psi_{p}^{\prime} .
\end{aligned}
$$

We apply an induction argument to prove the existence of solutions of $65-67$.

LEMma 3.2. Under the assumptions of Theorem 1.1, i.e., $P \in C^{4}(0, \infty), \mathcal{C} \in H^{2},\left(\omega_{1}, j_{1}\right)$ $\in H^{6} \times H^{5}$ with $\omega_{1}>0$ in $(0,1)$ and, for some $\alpha \in\left[(1+2 \sqrt{2} \varepsilon)^{-1}, 1\right)$,

$$
\left\|v_{1}\right\|_{C^{1}([0,1])}<\frac{(1-\alpha) \omega_{*}}{8 \sqrt{2}\left\|\omega_{1}\right\|_{1}}
$$

with

$$
\omega_{*}=\min _{x \in[0,1]} \omega_{1}(x)
$$

there exists a sequence $\left\{U^{i}\right\}_{i=1}^{\infty}$ of solutions of $65-67$ in the time interval $t \in\left[0, T_{*}\right]$ for some $T_{*}>0$ which is independent of $i$, satisfying the regularity properties

$$
\left\{\begin{array}{l}
\eta_{i} \in C^{1}\left(\left[0, T_{*}\right] ; H^{3}\right) \cap C^{2}\left(\left[0, T_{*}\right] ; H^{1}\right), \quad e_{i} \in C^{1}\left(\left[0, T_{*}\right] ; H^{4} \cap H_{0}^{1}\right), \\
\psi_{i} \in C^{l}\left(\left[0, T_{*}\right] ; H^{6-2 l} \cap H_{0}^{2}\right) \cap C^{3}\left(\left[0, T_{*}\right] ; L^{2}\right), \quad l=0,1,2, i \in \mathbb{N}
\end{array}\right.
$$


and the uniform bounds

$$
\left\{\begin{array}{l}
\left\|\eta_{i}^{\prime}(t)\right\|_{3}^{2}+\left\|\eta_{i}^{\prime \prime}(t)\right\|_{1}^{2}+\left\|\left(e_{i}, e_{i}^{\prime}\right)(t)\right\|_{4}^{2} \leq M_{0}, \\
\left\|\left(\psi_{i}, \psi_{i}^{\prime}, \psi_{i}^{\prime \prime}, \psi_{i}^{\prime \prime \prime}\right)(t)\right\|_{H^{6} \times H^{4} \times H^{2} \times L^{2}}^{2} \leq M_{0}, \quad i \geq 1, \quad t \in\left[0, T_{*}\right], \\
\left\|\eta_{i}(t)\right\|_{3}^{2} \leq 1, \quad\left\|\partial_{x}^{2} \psi_{i}(t)\right\|^{2} \leq \alpha^{2} \omega_{*}^{2},
\end{array}\right.
$$

where $M_{0}>0$ is a constant independent of $U^{i}(i \geq 1)$ and $T_{*}$.

Proof. Step 1: solution of 65-67 for $p \geq 1$. Obviously, $U^{1}=(0,0,0)$ satisfies 71-72. Starting with $U^{1}=(0,0,0)$, we prove the existence of a solution $U^{2}=\left(\psi_{2}, \eta_{2}, e_{2}\right)$ of 65-67 satisfying 71-72. The functions $g_{3}\left(x, U^{1}\right), g_{4}\left(x, U^{1}\right)$ and $k\left(x, U^{1}\right)$ only depend on the initial state $\left(\omega_{1}, j_{1}, \phi_{1}\right)$ and satisfy

$$
\begin{gathered}
g_{3}\left(x, U^{1}\right)=: \tilde{g}_{3}(x) \in H^{3}, \quad g_{4}\left(x, U^{1}\right)=: \tilde{g}_{4}(x) \in H^{2}, \quad k\left(x, U^{1}\right)=: \tilde{k}(x) \in H^{3}, \\
\partial_{t} g_{3}=\partial_{t} g_{4}=\partial_{t} k=0, \quad\left\|\tilde{g}_{3}\right\|_{3}^{2}+\left\|\tilde{g}_{4}\right\|_{2}^{2}+\|\tilde{k}\|_{3}^{2} \leq a_{0}\left(I_{0}+1\right),
\end{gathered}
$$

where $a_{0}>0$ is some constant and

$$
I_{0}=\left\|\left(\omega_{1}-\sqrt{\mathcal{C}}\right)\right\|^{2}+\left\|\omega_{1 x}\right\|_{5}^{2}+\left\|j_{1}\right\|_{5}^{2} .
$$

The existence of a solution $U^{2}=\left(\psi_{2}, \eta_{2}, e_{2}\right)$ of the linear system $65-67$ follows from the theory of ordinary differential equations, applied to 65 , Theorem 3.1 with $f(x, t)=\tilde{g}_{4}(x)$ and $b(x, t)=\tilde{k}(x)$, applied to 66 , and elliptic theory, applied to 67 . The solution $U^{2}$ exists on any time interval $[0, T], T>0$, and satisfies 71 with $T_{*}=T$ and the first two inequalities of (72) with $i=2$.

We show in the following that $U^{2}$ satisfies the last two inequalities of (72) for $t \in\left[0, T_{1}\right]$, where $T_{1}>0$ is given by

$$
T_{1}=\min \left\{\frac{\ln 2}{2+a_{0}\left(I_{0}+1\right)}, \frac{\nu \alpha^{2} \omega_{*}^{2}-4\left\|v_{1}\right\|_{C^{1}([0,1])}^{2}\left\|\omega_{1}\right\|_{1}^{2}}{2 a_{0}\left(I_{0}+1\right)}, \frac{1}{a_{0}\left(I_{0}+1\right)}\right\} .
$$

We recall that $a_{0}>0$ is a constant and $I_{0}$ and $\omega_{*}$ are given by 74 and 70 , respectively. It holds

$$
\nu \alpha^{2} \omega_{*}^{2}-4\left\|v_{1}\right\|_{C^{1}([0,1])}^{2}\left\|\omega_{1}\right\|_{1}^{2}>0
$$

since 69 implies

$$
4\left\|v_{1}\right\|_{C^{1}([0,1])}^{2}\left\|\omega_{1}\right\|_{1}^{2}<\frac{(1-\alpha)^{2} \omega_{*}^{2}}{32} \leq \nu \alpha^{2} \omega_{*}^{2} .
$$

From 65 we obtain by integrating

$$
\eta_{2}(t)=\tilde{g}_{3}(x) \int_{0}^{t} \exp \{-(t-s)\} d s, \quad t \in\left[0, T_{1}\right]
$$

and hence, in view of 75 ,

$$
\left\|\eta_{2}(t)\right\|_{3}^{2} \leq T_{1}^{2}\left\|\tilde{g}_{3}\right\|_{3}^{2} \leq 1, \quad t \in\left[0, T_{1}\right] .
$$


Multiplying the differential equation in (66) by $\psi_{2}^{\prime}$, integrating the resulting equation over $(0,1) \times(0, t)$ for $t \in\left[0, T_{1}\right]$, and integrating by parts gives

$$
\begin{aligned}
\left\|\psi_{2 x x}(t)\right\|^{2} & \leq \frac{1}{\nu}\left(\left\|\theta_{1}\right\|^{2}+a_{0} T_{1}\left(I_{0}+1\right)\right) e^{T_{1}\left(2+a_{0}\left(I_{0}+1\right)\right)} \\
& \leq \frac{2}{\nu}\left(2\left\|v_{1}\right\|_{C^{1}([0,1])}^{2}\left\|\omega_{1}\right\|_{1}^{2}+a_{0} T_{1}\left(I_{0}+1\right)\right) \\
& \leq \alpha^{2} \omega_{*}^{2}
\end{aligned}
$$

where we have used

$$
\left\|\theta_{1}\right\|^{2}=\left\|\omega_{1 x} v_{1}+\frac{1}{2} \omega_{1} v_{1 x}\right\|^{2} \leq 2\left\|v_{1}\right\|_{C^{1}([0,1])}^{2}\left\|\omega_{1}\right\|_{1}^{2} .
$$

This proves the last two bounds in (72). Moreover, by the Sobolev embedding theorem, it follows from 78 that

$$
\left\|\psi_{2}(t)\right\|_{1}^{2} \leq 2 \alpha^{2} \omega_{*}^{2}, \quad t \in\left[0, T_{1}\right] .
$$

Now, assume that there exist solutions $\left\{U^{i}\right\}_{i=1}^{p}(p \geq 2)$ of $65-67$ on the time interval $\left[0, T_{1}\right]$ where $T_{1}$ is given by 75 , satisfying 71-72. As above we obtain, for given $U^{p}$, the existence of a solution $U^{p+1}=\left(\psi_{p+1}, \eta_{p+1}, e_{p+1}\right)$ of $65-67$ in the interval $\left[0, T_{1}\right]$, satisfying

$$
\begin{aligned}
& \eta_{p+1} \in C^{1}\left(\left[0, T_{1}\right] ; H^{3}\right) \cap C^{2}\left(\left[0, T_{1}\right] ; H^{1}\right), \quad e_{p+1} \in C^{1}\left(\left[0, T_{1}\right] ; H^{4} \cap H_{0}^{1}\right), \\
& \psi_{p+1} \in C^{l}\left(\left[0, T_{1}\right] ; H^{6-2 l} \cap H_{0}^{2}\right) \cap C^{3}\left(\left[0, T_{1}\right] ; L^{2}\right), \quad l=0,1,2 .
\end{aligned}
$$

We prove that there exist constants $T_{*} \in\left(0, T_{1}\right]$ and $K_{i}>a_{0}(i=1,2,3,5,6,7)$ independent of $\left\{U^{i}\right\}_{i=1}^{p}$, such that if $U^{p}$ satisfies on $\left[0, T_{*}\right]$

$$
\begin{gathered}
\left\|\partial_{x}^{2} \psi_{p}(t)\right\|^{2} \leq \alpha^{2} \omega_{*}^{2}, \\
\left\|\left(\psi_{p}^{\prime \prime}, \psi_{p}^{\prime}\right)(t)\right\|_{2}^{2}+\left\|\psi_{p}^{\prime \prime \prime}(t)\right\|^{2} \leq K_{0}, \\
\left\|\partial_{x}^{3} \psi_{p}^{\prime}(t)\right\|_{1}^{2} \leq K_{1}, \quad\left\|\partial_{x}^{3} \psi_{p}(t)\right\|_{1}^{2} \leq K_{2}, \quad\left\|\partial_{x}^{5} \psi_{p}(t)\right\|_{1}^{2} \leq K_{3}, \\
\left\|\eta_{p}(t)\right\|_{3}^{2} \leq 1, \quad\left\|\eta_{p}^{\prime}(t)\right\|^{2} \leq K_{5}, \quad\left\|\partial_{x} \eta_{p}^{\prime}(t)\right\|_{2}^{2} \leq K_{6}, \quad\left\|\eta_{p}^{\prime \prime}(t)\right\|_{1}^{2} \leq K_{7},
\end{gathered}
$$

then $U^{p+1}$ also satisfies on $\left[0, T_{*}\right]$

$$
\begin{gathered}
\left\|\partial_{x}^{2} \psi_{p+1}(t)\right\|_{2}^{2} \leq \alpha^{2} \omega_{*}^{2}, \\
\left\|\left(\psi_{p+1}^{\prime \prime}, \psi_{p+1}^{\prime}\right)(t)\right\|_{2}^{2}+\left\|\psi_{p+1}^{\prime \prime \prime}(t)\right\|^{2} \leq K_{0}, \\
\left\|\partial_{x}^{3} \psi_{p+1}^{\prime}(t)\right\|_{1}^{2} \leq K_{1}, \quad\left\|\partial_{x}^{3} \psi_{p+1}(t)\right\|_{1}^{2} \leq K_{2}, \quad\left\|\partial_{x}^{5} \psi_{p+1}(t)\right\|_{1}^{2} \leq K_{3}, \\
\left\|\eta_{p+1}(t)\right\|_{3}^{2} \leq 1, \quad\left\|\eta_{p+1}^{\prime}(t)\right\|^{2} \leq K_{5}, \quad\left\|\partial_{x} \eta_{p+1}^{\prime}(t)\right\|_{2}^{2} \leq K_{6}, \quad\left\|\eta_{p+1}^{\prime \prime}(t)\right\|_{1}^{2} \leq K_{7} .
\end{gathered}
$$

Notice that it follows from 81 and 85, employing the boundary conditions in (66) and Poincaré's inequality,

$$
\left\|\psi_{p}(t)\right\|_{1}^{2} \leq 2 \alpha^{2} \omega_{*}^{2}, \quad\left\|\psi_{p+1}(t)\right\|_{1}^{2} \leq 2 \alpha^{2} \omega_{*}^{2}, \quad t \in\left[0, T_{*}\right]
$$


Step 2: estimates for $g_{3}, g_{4}$, and $k$. Let $U^{p}$ satisfy 81-84. Then a direct computation shows the following estimates for $g_{3}\left(x, U^{p}\right)$ and $g_{4}\left(x, U^{p}\right)$, for $t \in\left[0, T_{*}\right]$ :

$$
\begin{aligned}
\left\|g_{3}\left(\cdot, U^{p}\right)(t)\right\|_{1}^{2} \leq & N\left(I_{0}+1+\left\|\psi_{p}(t)\right\|_{4}^{2}+\left\|\psi_{p}^{\prime}(t)\right\|_{1}^{2}\right)^{5} \\
\leq & N\left(I_{0}+1+K_{0}+K_{2}\right)^{5} \\
\left\|g_{3 x}\left(\cdot, U^{p}\right)(t)\right\|_{2}^{2} \leq & N\left(I_{0}+1+\left\|\psi_{p}(t)\right\|_{6}^{2}+\left\|\psi_{p}^{\prime}(t)\right\|_{3}^{2}\right)^{7} \\
\leq & N\left(I_{0}+1+K_{0}+K_{1}+K_{2}+K_{3}\right)^{7} \\
\left\|g_{3}^{\prime}\left(\cdot, U^{p}\right)(t)\right\|_{1}^{2} \leq & N\left(I_{0}+1+\left\|\eta_{p}^{\prime}(t)\right\|_{1}^{2}+\left\|\psi_{p}^{\prime}(t)\right\|_{4}+\left\|\psi_{p}(t)\right\|_{4}^{2}+\left\|\psi_{p}^{\prime \prime}(t)\right\|_{1}^{2}\right)^{6} \\
\leq & N\left(I_{0}+1+K_{0}+K_{1}+K_{2}+K_{3}+K_{5}+K_{6}\right)^{6} \\
\left\|g_{4}\left(\cdot, U^{p}\right)(t)\right\|^{2} \leq & N\left(I_{0}+1+\left\|\psi_{p}(t)\right\|_{4}^{2}+\left\|\psi_{p}^{\prime}(t)\right\|_{1}^{2}\right)^{3} \\
& +16 \nu^{2} a_{1}\left\|\partial_{x}^{2} \psi_{p}(t)\right\|^{2}\left\|\partial_{x}^{4} \psi_{p}(t)\right\|^{2} \\
\leq & N\left(I_{0}+1+K_{0}+K_{2}\right)^{3} \\
\left\|g_{4 x}\left(\cdot, U^{p}\right)(t)\right\|_{1}^{2} \leq & N\left(I_{0}+1+\left\|\psi_{p}(t)\right\|_{4}^{2}+\left\|\psi_{p}^{\prime}(t)\right\|_{2}^{2}\right)^{5} \\
\leq & N\left(I_{0}+1+K_{0}+K_{2}\right)^{5} \\
\left\|g_{4}^{\prime}\left(\cdot, U^{p}\right)(t)\right\|^{2} \leq & N\left(I_{0}+1+\left\|\eta_{p}^{\prime}(t)\right\|^{2}+\left\|\psi_{p}(t)\right\|_{3}^{2}+\left\|\psi_{p}^{\prime}(t)\right\|_{2}^{2}+\left\|\psi_{p}^{\prime \prime}(t)\right\|^{2}\right)^{4} \\
\leq & N\left(I_{0}+1+K_{0}+K_{2}+K_{5}\right)^{4} \\
\left\|g_{4}^{\prime \prime}\left(\cdot, U^{p}\right)(t)\right\|^{2} \leq & N\left(I_{0}+1+\left\|\left(\eta_{p}^{\prime}, \eta_{p}^{\prime \prime}, \psi_{p}^{\prime \prime \prime}\right)(t)\right\|^{2}+\left\|\left(\psi_{p}, \psi_{p}^{\prime}\right)(t)\right\|_{3}^{2}+\left\|\psi_{p}^{\prime \prime}(t)\right\|_{2}^{2}\right)^{5} \\
\leq & N\left(I_{0}+1+K_{0}+K_{1}+K_{2}+K_{5}+K_{7}\right)^{5}
\end{aligned}
$$

where

$$
a_{1}=\max _{x \in[0,1]}\left(\omega_{1}+\psi_{p}\right)^{-2}=(1-\alpha)^{-2} \omega_{*}^{-2},
$$

and the estimates for $k\left(x, U^{p}\right)$ and $e_{p}(x, t)$, for $t \in\left[0, T_{*}\right]$,

$$
\begin{aligned}
\left\|k\left(\cdot, U^{p}\right)(t)\right\|_{2}^{2} & \leq N\left(I_{0}+1+\left\|\psi_{p}(t)\right\|_{2}^{2}\right)^{3} \\
& \leq N\left(I_{0}+1\right)^{3} \\
\left\|k^{\prime}\left(\cdot, U^{p}\right)(t)\right\|^{2} & \leq N\left(I_{0}+1\right)\left(\left\|\eta_{p}^{\prime}(t)\right\|^{2}+\left\|\psi_{p}^{\prime}(t)\right\|^{2}\right) \\
& \leq N\left(I_{0}+1+K_{0}+K_{5}\right)^{2} \\
\left\|k^{\prime \prime}\left(\cdot, U^{p}\right)(t)\right\|^{2} & \leq N\left(I_{0}+1\right)\left(\left\|\left(\eta_{p}^{\prime \prime}, \eta_{p}^{\prime}, \psi_{p}^{\prime \prime}\right)(t)\right\|^{2}+\left\|\psi_{p}^{\prime}(t)\right\|_{1}^{2}\right)^{2} \\
& \leq N\left(I_{0}+1+K_{0}+K_{5}+K_{7}\right)^{3} \\
\left\|e_{p}\right\|_{4}^{2}+\left\|\left(e_{p}^{\prime}, e_{p}^{\prime \prime}\right)\right\|_{2}^{2} & \leq N\left(\left\|\psi_{p}(t)\right\|_{4}^{2}+\left\|\left(\psi_{p}^{\prime}, \psi_{p}^{\prime \prime}\right)(t)\right\|_{2}^{2}\right) \\
& \leq N\left(I_{0}+1+K_{0}+K_{2}\right),
\end{aligned}
$$

where $N>1$ is a constant independent of $K_{i}(i=1,2,3,5,6,7)$.

Step 3: estimates for $\eta_{p+1}$. Integration of 65 yields

$$
\eta_{p+1}(x, t)=\int_{0}^{t} \exp \{-(t-s)\} g_{3}\left(x, U^{1}\right)(s) d s, \quad 0 \leq t \leq T_{*} \leq T_{1}, \quad x \in[0,1],
$$

and

$$
\eta_{p+1} \in C^{1}\left(\left[0, T_{1}\right] ; H^{3}\right) \cap C^{2}\left(\left[0, T_{*}\right] ; H^{1}\right)
$$


From $90-92$ we obtain the estimates

$$
\begin{aligned}
\left\|\eta_{p+1}(t)\right\|^{2} & \leq T_{*}^{2}\left\|g_{3}\left(\cdot, U^{p}\right)\right\|^{2} \leq T_{*}^{2} N\left(I_{0}+1+K_{0}+K_{2}\right)^{5} \\
\left\|\partial_{x} \eta_{p+1}(t)\right\|_{2}^{2} & \leq T_{*}^{2}\left\|g_{3 x}\left(\cdot, U^{p}\right)\right\|_{2}^{2} \leq T_{*}^{2} N\left(I_{0}+1+K_{0}+K_{1}+K_{2}+K_{3}\right)^{7} \\
\left\|\eta_{p+1}^{\prime}(t)\right\|^{2} & \leq 2\left(T_{1}^{2}+1\right)\left\|g_{3}\left(\cdot, U^{p}\right)\right\|^{2} \leq 2 N\left(T_{1}^{2}+1\right)\left(I_{0}+1+K_{0}+K_{2}\right)^{5} \\
\left\|\partial_{x} \eta_{p+1}^{\prime}(t)\right\|_{2}^{2} & \leq 2\left(T_{1}^{2}+1\right)\left\|g_{3 x}\left(\cdot, U^{p}\right)\right\|_{2}^{2} \\
& \leq 2 N\left(T_{1}^{2}+1\right)\left(I_{0}+1+K_{0}+K_{1}+K_{2}+K_{3}\right)^{7} \\
\left\|\eta_{p+1}^{\prime \prime}(t)\right\|_{1} \leq & 4\left(T_{1}^{2}+1\right)\left\|g_{3}\left(\cdot, U^{p}\right)\right\|_{1}^{2}+2\left\|g_{3}^{\prime}\left(\cdot, U^{p}\right)\right\|_{1}^{2} \\
& \leq 4 N\left(T_{1}^{2}+1\right)\left(I_{0}+1+K_{0}+K_{1}+K_{2}+K_{3}\right)^{7} \\
& +2 N\left(I_{0}+1+K_{0}+K_{1}+K_{2}+K_{3}+K_{5}+K_{6}\right)^{6} .
\end{aligned}
$$

Thus, $\eta_{p+1}$ satisfies 88 if

$$
\begin{aligned}
K_{5}= & 2 N\left(T_{1}^{2}+1\right)\left(I_{0}+1+K_{0}+K_{2}\right)^{5} \\
K_{6}= & 2 N\left(T_{1}^{2}+1\right)\left(I_{0}+1+K_{0}+K_{1}+K_{2}+K_{3}\right)^{7}, \\
K_{7}= & 2 N\left(T_{1}^{2}+1\right)\left(I_{0}+1+K_{0}+K_{1}+K_{2}+K_{3}\right)^{7} \\
& +2 N\left(I_{0}+1+K_{0}+K_{1}+K_{2}+K_{3}+K_{5}+K_{6}\right)^{6}
\end{aligned}
$$

and if $T_{*}$ satisfies

$$
T_{*} \leq \frac{1}{\sqrt{L_{1}}}
$$

where

$$
L_{1}=\min \left\{2 N\left(I_{0}+1+K_{0}+K_{2}\right)^{5}, 2 N\left(I_{0}+1+K_{0}+K_{1}+K_{2}+K_{3}\right)^{7}\right\} .
$$

Step 4: estimates for $\psi_{p+1}$. We multiply the differential equation in 66 by $\psi_{p+1}^{\prime}$, $\psi_{p+1}^{\prime \prime}$, and $\psi_{p+1}^{\prime \prime \prime}$, respectively, integrate the sum of the resulting equations over $(0,1) \times$ $\left(0, T_{*}\right)$ and integrate by parts. In view of $93-94,96-97,99-101$, we obtain after tedious computations

$$
\begin{aligned}
\left\|\partial_{x}^{2} \psi_{p+1}(t)\right\|^{2} & \leq \frac{1}{\nu}\left(\left\|\theta_{1}\right\|_{2}^{2}+T_{*}\left\|g_{4}\left(\cdot, U^{p}\right)\right\|^{2}\right) e^{T_{*}\left(2+N\left(I_{0}+1\right)^{2}\right)} \\
& \leq \frac{1}{\nu}\left(2\left\|v_{1}\right\|_{C^{1}([0,1])}^{2}\left\|\omega_{1}\right\|_{1}^{2}+T_{*} L_{3}\right) e^{T_{*}\left(2+N\left(I_{0}+1\right)^{2}\right)}
\end{aligned}
$$

and

$$
\begin{aligned}
& \left\|\psi_{p+1}^{\prime \prime \prime}(t)\right\|^{2}+\left\|\psi_{2}^{\prime \prime}(t)\right\|^{2}+\nu\left\|\partial_{x}^{2} \psi_{p+1}^{\prime \prime}(t)\right\|^{2}+\nu\left\|\partial_{x}^{2} \psi_{p+1}^{\prime}(t)\right\|^{2}+\left\|\psi_{p+1}^{\prime}\right\|^{2} \\
\leq & \left(\left\|\psi_{p+1}^{\prime \prime \prime}(0)\right\|^{2}+2\left\|\psi_{2}^{\prime \prime}(0)\right\|+\nu\left\|\partial_{x}^{2} \psi_{p+1}^{\prime \prime}(0)\right\|^{2}+\left\|\theta_{1}\right\|^{2}+\nu\left\|\partial_{x}^{2} \theta_{1}\right\|^{2}\right) e^{T_{*} L_{4}} \\
& +T_{*}\left(\left\|g_{4}^{\prime \prime}\left(\cdot, U^{p}\right)\right\|^{2}+\left\|g_{4}^{\prime}\left(\cdot, U^{p}\right)\right\|^{2}\right) e^{T_{*} L_{4}} \\
\leq & N\left(I_{0}+T_{*} L_{5}\right) e^{T_{*} L_{4}}
\end{aligned}
$$


where

$$
\begin{aligned}
& L_{3}=N\left(I_{0}+1+K_{0}+K_{2}\right)^{3}, \\
& L_{4}=8+6 N\left(I_{0}+1+K_{0}+K_{5}+K_{7}\right)^{3}>2+N\left(I_{0}+1\right)^{2}, \\
& L_{5}=2 N\left(I_{0}+1+K_{0}+K_{1}+K_{2}+K_{5}+K_{7}\right)^{5} .
\end{aligned}
$$

Define

$$
K_{0}=20 N I_{0} \cdot \frac{1}{\min \{1, \nu\}}=20 N I_{0} \cdot \max \left\{1, \nu^{-1}\right\} .
$$

Using 76 (which is a consequence of 69 ), we see that $\psi_{p+1}$ satisfies

$$
\begin{gathered}
\left\|\partial_{x}^{2} \psi_{p+1}(t)\right\|^{2} \leq \alpha^{2} \omega_{*}^{2}, \\
\left\|\left(\psi_{p+1}^{\prime}, \psi_{p+1}^{\prime \prime}\right)(t)\right\|_{2}^{2}+\left\|\psi_{p+1}^{\prime \prime \prime}(t)\right\|^{2} \leq 20 N I_{0} \cdot \max \left\{1, \nu^{-1}\right\}=K_{0}
\end{gathered}
$$

if

$$
T_{*} \leq \min \left\{\frac{1}{\sqrt{L_{1}}}, \frac{B_{1}}{4 L_{3}}, \frac{\ln 2}{2+N\left(I_{0}+1\right)^{2}}, \frac{\ln 2}{2+N\left(I_{0}+1\right)}, \frac{\ln 2}{L_{4}}, \frac{I_{0}}{L_{5}}\right\},
$$

where

$$
B_{1}=\nu \alpha^{2} \omega_{*}^{2}-4\left\|v_{1}\right\|_{C^{1}([0,1])}^{2}\left\|\omega_{1}\right\|_{1}^{2}
$$

This proves 85 and 86 .

To verify 87 we employ the differential equation in (66) again. We use 116, 93, 79, and 117 to estimate

$$
\begin{aligned}
\left\|\partial_{x}^{4} \psi_{p+1}(t)\right\|^{2} \leq & \frac{N}{\nu^{2}}\left(\left\|\psi_{p+1}^{\prime \prime}(t)\right\|^{2}+\left\|\psi_{p+1}^{\prime}(t)\right\|^{2}+\left\|\psi_{p+1}(t)\right\|^{2}+\left\|k \partial_{x} \psi_{p+1}^{\prime}(t)\right\|^{2}\right) \\
& +\frac{2}{\nu^{2}}\left\|g_{4}\left(\cdot, U^{p}\right)(t)\right\|^{2} \\
\leq & \frac{2 N}{\nu^{2}}\left(I_{0}+1\right)^{3}\left(1+K_{0}\right)+32 a_{1}\left\|\partial_{x}^{2} \psi_{p}(t)\right\|^{2} K_{2} \\
\leq & \frac{2 N}{\nu^{2}}\left(I_{0}+1\right)^{3}\left(1+K_{0}\right)+32 a_{1} e^{T_{*}\left(2+N\left(I_{0}+1\right)^{2}\right)}\left(\left\|\theta_{1}\right\|_{2}^{2}+T_{*} L_{3}\right) K_{2} \\
\leq & \frac{2 N}{\nu^{2}}\left(I_{0}+1\right)^{3}\left(1+K_{0}\right)+64 a_{1}\left(2\left\|v_{1}\right\|_{C^{1}([0,1])}^{2}\left\|\omega_{1}\right\|_{1}^{2}+T_{*} L_{3}\right) K_{2} .
\end{aligned}
$$

Here, we used the fact that 110 is also valid for $\psi_{p}$ in $\left[0, T_{*}\right]$.

From 69 and 98 we infer

$$
128 a_{1}\left\|v_{1}\right\|_{C^{1}([0,1])}^{2}\left\|\omega_{1}\right\|_{1}^{2}=128\left\|v_{1}\right\|_{C^{1}([0,1])}^{2}(1-\alpha)^{-2} \omega_{*}^{-2}\left\|\omega_{1}\right\|_{1}^{2}<1,
$$

which implies

$$
1-\frac{128\left\|v_{1}\right\|_{C^{1}([0,1])}^{2}\left\|\omega_{1}\right\|_{1}^{2}}{(1-\alpha)^{2} \omega_{*}^{2}}>0
$$

Thus, choosing

$$
K_{2}=\frac{8(1-\alpha)^{2} \omega_{*}^{2} N\left(I_{0}+1\right)^{3}\left(1+K_{0}\right)}{\nu^{2}\left[(1-\alpha)^{2} \omega_{*}^{2}-128\left\|v_{1}\right\|_{C^{1}([0,1])}^{2}\left\|\omega_{1}\right\|_{1}^{2}\right]},
$$

where $K_{0}$ is defined by 115 , we obtain from 118 and the Sobolev embedding theorem

$$
\left\|\partial_{x}^{3} \psi_{p+1}(t)\right\|^{2}+\left\|\partial_{x}^{4} \psi_{p+1}(t)\right\|^{2} \leq K_{2}
$$


if

$$
T_{*}=\min \left\{\frac{1}{\sqrt{L_{1}}}, \frac{B_{0}}{L_{3}}, \frac{\ln 2}{2+N\left(I_{0}+1\right)}, \frac{\ln 2}{L_{4}}, \frac{I_{0}}{L_{5}}\right\} .
$$

We recall that $L_{1}, L_{3}, L_{4}, L_{5}$, and $I_{0}$ are given by $109,112-114$, and 74, respectively, $N>0$ is a generic constant, and

$$
B_{0}=\min \left\{\frac{(1-\alpha)^{2} \omega_{*}^{2}-128\left\|v_{1}\right\|_{C^{1}([0,1])}^{2}\left\|\omega_{1}\right\|_{1}^{2}}{64}, \frac{\alpha^{2} \omega_{*}^{2} \varepsilon^{2}-16\left\|v_{1}\right\|_{C^{1}([0,1])}^{2}\left\|\omega_{1}\right\|_{1}^{2}}{8}\right\}>0
$$

due to 69 . Notice that 120 implies 108 and 117.

Differentiating the differential equation in (66) with respect to $t$, integrating over $(0,1)$, and using 116,99 , and 95 , we can estimate $\partial_{x}^{4} \psi_{p+1}^{\prime}$ as

$$
\begin{aligned}
\left\|\partial_{x}^{4} \psi_{p+1}^{\prime}(t)\right\|^{2} \leq & \frac{N}{\nu^{2}}\left(\left\|\psi_{p+1}^{\prime \prime \prime}(t)\right\|^{2}+\left\|\psi_{p+1}^{\prime \prime}(t)\right\|^{2}+\left\|\psi_{p+1}^{\prime}(t)\right\|^{2}+\left\|\left(k \partial_{x} \psi_{p+1}^{\prime}\right)^{\prime}(t)\right\|^{2}\right) \\
& +\frac{2}{\nu^{2}}\left\|g_{4}^{\prime}\left(\cdot, U^{p}\right)(t)\right\|^{2} \\
\leq & \frac{2 N}{\nu^{2}}\left(I_{0}+1+K_{0}+K_{2}+K_{5}\right)^{4} .
\end{aligned}
$$

Thus, by the Sobolev embedding theorem, we have

$$
\left\|\partial_{x}^{3} \psi_{p+1}^{\prime}(t)\right\|^{2}+\left\|\partial_{x}^{4} \psi_{p+1}^{\prime}(t)\right\|^{2} \leq K_{1}
$$

if

$$
K_{1}=\frac{4 N}{\nu^{2}}\left(I_{0}+1+K_{0}+K_{2}+K_{5}\right)^{4}
$$

Differentiating the differential equation in (66) once and twice with respect to $x$, integrating over $(0,1)$, and employing 116,99 , and 95 , we can estimate $\partial_{x}^{5} \psi_{p+1}$ and $\partial_{x}^{6} \psi_{p+1}$ as

$$
\begin{aligned}
\left\|\partial_{x}^{5} \psi_{p+1}(t)\right\|^{2} \leq & \frac{N}{\nu^{2}}\left(\left\|\partial_{x} \psi_{p+1}^{\prime \prime}(t)\right\|^{2}+\left\|\partial_{x} \psi_{p+1}^{\prime}(t)\right\|^{2}+\left\|\partial_{x} \psi_{p+1}(t)\right\|^{2}\right) \\
& +\frac{N}{\nu^{2}}\left\|\partial_{x}\left(k \partial_{x} \psi_{p+1}^{\prime}\right)(t)\right\|^{2}+\frac{2}{\nu^{2}}\left\|\partial_{x} g_{4}\left(\cdot, U^{2} p\right)(t)\right\|^{2} \\
\leq & \frac{1}{2} K_{3}, \\
\left\|\partial_{x}^{6} \psi_{p+1}(t)\right\|^{2} \leq & \frac{N}{\nu^{2}}\left(\left\|\partial_{x}^{2} \psi_{p+1}^{\prime \prime}(t)\right\|^{2}+\left\|\partial_{x}^{2} \psi_{p+1}^{\prime}(t)\right\|^{2}+\left\|\partial_{x}^{2} \psi_{p+1}(t)\right\|^{2}\right) \\
& +\frac{N}{\nu^{2}}\left\|\partial_{x}^{2}\left(k \partial_{x} \psi_{p+1}^{\prime}\right)(t)\right\|^{2}+\frac{2}{\nu^{2}}\left\|\partial_{x}^{2} g_{4}\left(\cdot, U^{2}\right)(t)\right\|^{2} \\
\leq & \frac{1}{2} K_{3},
\end{aligned}
$$

if we choose

$$
K_{3}=\frac{6 N}{\nu^{2}}\left(I_{0}+1+K_{0}+K_{1}+K_{2}\right)^{5}
$$

Now we choose the constants $K_{i}$ as follows. Let $K_{0}$ be given by $115, K_{2}$ by $119, K_{5}$ by $105, K_{1}$ by $122, K_{3}$ by $125, K_{6}$ by 106 , and $K_{7}$ by 107 (with $\nu=\varepsilon^{2} / 4$ ). The constant $T_{*}$ is determined by 120 . This shows that $\left(\psi_{p+1}, \eta_{p+1}\right)$ satisfies $85-88$ for $t \in\left[0, T_{*}\right]$. 
Step 5: end of the proof. The uniform bounds for $e_{p+1} \in C^{1}\left(\left[0, T_{*}\right] ; H^{4}\right)$ of 67 follow from similar computations as those needed to derive 102, where the index $p$ is replaced by $p+1$.

By induction, we conclude that $\left\{U^{i}\right\}_{p=1}^{\infty}$ exists uniformly in $\left[0, T_{*}\right]$ with $T_{*}$ given by 120 and satisfies 71-72 uniformly for

$$
M_{0}=\max \left\{K_{0}, K_{1}, K_{2}, K_{3}, K_{5}, K_{6}, K_{7}\right\} .
$$

The proof of Lemma 3.2 is complete.

REMARK 3.3. It follows from the last two inequalities of (72) that

$$
\psi_{i}(x, t)+\omega_{1}(x) \geq(1-\alpha) \omega_{*}>0, \quad i \geq 1
$$

For the proof of Theorem 1.1, we observe that after a tedious computation similarly as in the proof of Lemma 3.2, we can obtain the following estimates:

$$
\begin{aligned}
& \left\|\eta_{p+1}-\eta_{p}\right\|_{C^{1}\left(0, T_{* *} ; H^{1}\right)}^{2}+\left\|\psi_{p+1}-\psi_{p}\right\|_{C^{i}\left(0, T_{* *} ; H^{4-2 i}\right)}^{2}+\left\|e_{p+1}-e_{p}\right\|_{C^{1}\left(0, T_{* *} ; H^{2}\right)}^{2} \\
\leq & T_{* *} \alpha\left(N, M_{0}\right)\left(\left\|\eta_{p}-\eta_{p-1}\right\|_{C^{1}\left(0, T_{* *} ; H^{1}\right)}^{2}+\left\|\psi_{p}-\psi_{p-1}\right\|_{C^{i}\left(0, T_{* *} ; H^{4-2 i}\right)}^{2}\right), i=0,1,2,
\end{aligned}
$$

for any $T_{* *} \leq T_{*}$. Here $\alpha\left(N, M_{0}\right)$ is a function of $N, M_{0}$. Taking $T_{* *}$ such that

$$
T_{* *}<\min \left\{\frac{b_{1}}{\alpha\left(N, M_{0}\right)}, \quad T_{*}\right\}, \quad b_{1} \in(0,1),
$$

then it follows from 127 :

$$
\begin{aligned}
& \sum_{p=1}^{\infty}\left(\left\|\eta_{p+1}-\eta_{p}\right\|_{C^{1}\left(0, T_{* *} ; H^{1}\right)}^{2}+\left\|e_{p+1}-e_{p}\right\|_{C^{1}\left(0, T_{* *} ; H^{2}\right)}^{2}\right) \\
& +\sum_{p=1}^{\infty}\left\|\psi_{p+1}-\psi_{p}\right\|_{C^{i}\left(0, T_{* *} ; H^{4-2 i}\right)}^{2} \leq C, \quad i=0,1,2,
\end{aligned}
$$

with $C>0$ a constant.

Proof of Theorem 1.1. By Lemma 3.2 and 129, the sequence $\left\{U^{p}\right\}_{p=1}^{\infty}$ satisfies $71-72$, 126, and 129 uniformly in $[0, T]$ with $T \leq T_{* *}$. Applying the Ascoli-Arzela theorem and the Aubin-Lions lemma to $\left\{U^{p}\right\}_{p=1}^{\infty}$, it follows that there exists $U=(\psi, \eta, e)$ satisfying

$$
\begin{gathered}
\eta \in C^{1}\left([0, T] ; H^{3}\right), \quad e \in C^{1}\left([0, T] ; H^{4}\right), \\
\psi \in C^{i}\left([0, T] ; H^{6-2 i} \cap H_{0}^{2}\right) \cap C^{3}\left([0, T] ; L^{2}\right), \quad i=0,1,2,
\end{gathered}
$$

and there is a subsequence $\left\{U^{p_{j}}, U^{p_{j}+1}\right\}_{j=1}^{\infty}$ with $p_{j}+1 \leq p_{j+1}$ such that

$$
\begin{array}{ll}
\psi_{p_{j}+1}, \psi_{p_{j}} \stackrel{j \rightarrow \infty}{\longrightarrow} \psi & \text { strongly in } C^{i}\left([0, T] ; H^{6-2 i-\sigma}\right), i=0,1,2, \\
\eta_{p_{j}+1}, \eta_{p_{j}} \stackrel{j \rightarrow \infty}{\longrightarrow} \eta & \text { strongly in } C^{1}\left([0, T] ; H^{3-\sigma}\right), \\
e_{p_{j}+1}, e_{p_{j}} \stackrel{j \rightarrow \infty}{\longrightarrow} e & \text { strongly in } C^{1}\left([0, T] ; H^{4-\sigma}\right),
\end{array}
$$

for any $\sigma>0$. 
It is not difficult to verify that $U$ is a solution of $65-67$ and satisfies 126 where $U^{p}$ is replaced by $U$. Setting

$$
\omega=\omega_{1}+\psi>0, \quad j=j_{1}+\eta, \quad \phi=\phi_{1}+e,
$$

we see that $j \in C\left([0, T] ; H^{5}\right)$ and $(w, j, \phi)$ is a local-in-time solution of the IBVP 14-19. The uniqueness can be proven similarly as the estimates 129 . The proof of Theorem 1.1 is complete.

Acknowledgments. The first author acknowledges partial support from the TMR project "Asymptotic Methods in Kinetic Theory", grant ERB-FMBX-CT97-0157, from the Gerhard-Hess Award of the Deutsche Forschungsgemeinschaft (DFG), grant JU359/32, from the Priority Program "Multi-scale Problems" of the DFG, grant JU359/5-1, and from the AFF Project of the University of Konstanz, grant 4/00. The second author acknowledges partial support from the Austrian-Chinese Scientific-Technical Collaboration Agreement, from CTS of Taiwan, from the Wittgenstein Award 2000 of P. A. Markowich, funded by the Austrian FW, and from the Grants-in-Aid of Japan Society for the Promotion of Science for JSPS fellows No. 14-02036.

\section{REFERENCES}

[1] F. Brezzi, I. Gasser, P. Markowich, and C. Schmeiser, Thermal equilibrium state of the quantum hydrodynamic model for semiconductors in one dimension, Appl. Math. Lett. 8 (1995), 47-52.

[2] P. Degond and P. A. Markowich, On a one-dimensional steady-state hydrodynamic model, Appl. Math. Lett. 3 (1990), 25-29.

[3] K. O. Friedrichs, Symmetric positive linear differential equations, Comm. Pure Appl. Math. 11 (1958), 333-418.

[4] I. Gamba and A. Jüngel, Positive solutions to singular second and third order differential equations for quantum fluids, Arch. Rational Mech. Anal. 156 (2001), 183-203.

[5] C. Gardner, Numerical simulation of a steady-state electron shock wave in a submicron semiconductor device, IEEE Trans. El. Dev. 38 (1991), 392-398.

[6] C. Gardner, The quantum hydrodynamic model for semiconductors devices, SIAM J. Appl. Math. 54 (1994), 409-427.

[7] I. Gasser and A. Jüngel, The quantum hydrodynamic model for semiconductors in thermal equilibrium, Z. Angew. Math. Phys. 48 (1997), 45-59.

[8] I. Gasser and P. Mårkowich, Quantum hydrodynamics, Wigner transforms and the classical limit, Asymptotic Anal. 14 (1997), 97-116.

[9] I. Gasser, P. A. Markowich, and C. Ringhofer, Closure conditions for classical and quantum moment hierarchies in the small temperature limit, Transp. Theory Stat. Phys. 25 (1996), 409-423.

[10] M. T. Gyi and A. Jüngel, A quantum regularization of the one-dimensional hydrodynamic model for semiconductors, Adv. Diff. Eqs. 5 (2000), 773-800.

[11] A. Jüngel, A steady-state potential flow Euler-Poisson system for charged quantum fluids, Comm. Math. Phys. 194 (1998), 463-479.

[12] A. Jüngel, Quasi-hydrodynamic semiconductor equations, Progress in Nonlinear Differential Equations, Birkhäuser, Basel (2001).

[13] A. Jüngel, M. C. Mariani, and D. Rial, Local existence of solutions to the transient quantum hydrodynamic equations, Math. Models Meth. Appl. Sci. 12 (2002), 485-495.

[14] A. Jüngel and H.-L. Li, Quantum Euler-Poisson systems: existence of stationary states, to appear in Arch. Math. (2003).

[15] T. Kato, The Cauchy problem for quasi-linear symmetric hyperbolic systems, Arch. Rational Mech. Anal. 58 (1975), 181-205.

[16] L. D. Landau and E. M. Lifshitz, Quantum Mechanics: Non-relativistic Theory, New York, Pergamon Press (1977). 
[17] H.-L. Li and C.-K. Lin, Semiclassical limit and well-posedness of Schrödinger-Poisson system, Electronic Journal of Differential Equations, Vol. 2003 (2003), No. 93, 1-17.

[18] H.-L. Li and P. Marcati, Existence and asymptotic behavior of multi-dimensional quantum hydrodynamic model for semiconductors, preprint (2002).

[19] H.-L. Li, M. Mei, and P. A. Markowich, Asymptotic behavior of solutions of the hydrodynamic model of semiconductors, Proc. Roy. Soc. Edinburgh, Sect. A 132 (2002), no. 2, 359-378.

[20] M. Loffredo and L. Morato, On the creation of quantum vortex lines in rotating HeII, Il nouvo cimento 108B (1993), 205-215.

[21] E. Madelung, Quantentheorie in hydrodynamischer Form, Z. Physik 40 (1927), 322.

[22] A. Majda, Compressible Fluid Flow and Systems of Conservation Laws in Several Space Variables, Appl. Math. Sci. 53, Springer (1984).

[23] T. Makino and S. Ukai, Sur l'existence des solutions locales de l'équation d'Euler-Poisson pour l'évolution d'étoiles gazeuses, J. Math. Kyoto Univ. 27 (1987), 387-399.

[24] P. A. Markowich, C. Ringhofer, and C. Schmeiser, Semiconductor Equations, Springer, Wien (1990).

[25] C.-W. Shu, Essentially non-oscillatory and weighted essentially non-oscillatory schemes for hyperbolic conservation laws, ICASE Report No. 97-65, NASA Langley Research Center, Hampton, USA (1997).

[26] R. Temam, Infinite-dimensional Dynamical Systems in Mechanics and Physics, Appl. Math. Sci. 68, Springer (1988).

[27] B. Zhang and J. Jerome, On a steady-state quantum hydrodynamic model for semiconductors, Nonlinear Anal., TMA 26 (1996), 845-856.

[28] E. Zeidler, Nonlinear functional analysis and its applications. Vol. II: Nonlinear monotone operators, Springer (1990). 